\title{
A Wave Propagation Algorithm for Hyperbolic Systems on Curved Manifolds
}

\author{
James A. Rossmanith ${ }^{\mathrm{a}}$, Derek S. Bale ${ }^{\mathrm{b}}$, Randall J. LeVeque ${ }^{\mathrm{c}}$ \\ ${ }^{a}$ Department of Mathematics, University of Michigan, 525 East University Ave., \\ Ann Arbor, MI 48109-1109, USA \\ ${ }^{\mathrm{b}}$ Product Engineering, eV Products, a Division of II-VI Inc., 373 Saxonburg Blvd., \\ Saxonburg, PA 16056, USA \\ ${ }^{\mathrm{c}}$ Department of Applied Mathematics, University of Washington, Box 352420, \\ Seattle, WA 98195-2420, USA
}

\begin{abstract}
An extension of the wave propagation algorithm first introduced by LeVeque $[J$. Comp. Phys. 131, 327-353 (1997)] is developed for hyperbolic systems on a general curved manifold. This extension is important in a variety of applications, including the propagation of sound waves on a curved surface, shallow water flow on the surface of the Earth, shallow water magnetohydrodynamics in the solar tachocline, and relativistic hydrodynamics in the presence of compact objects such as neutron stars and black holes. As is the case for the Cartesian wave propagation algorithm, this new approach is second order accurate for smooth flows and high-resolution shockcapturing. The algorithm is formulated such that scalar variables are numerically conserved and vector variables have a geometric source term that is naturally incorporated into a modified Riemann solver. Furthermore, all necessary one-dimensional Riemann problems are solved in a locally valid orthonormal basis. This orthonormalization allows one to solve Cartesian Riemann problems that are devoid of geometric terms. The new method is tested via application to the linear wave equation on a curved manifold as well as the shallow water equations on part of a sphere. The proposed algorithm has been implemented in the software package CLAWPACK and is freely available on the web.
\end{abstract}

Key words: wave propagation algorithms, high-resolution methods, hyperbolic systems, curved manifolds

Email addresses: rossmani@umich.edu (James A. Rossmanith), dbale@ii-vi.com (Derek S. Bale), rjl@amath.washington.edu (Randall J. LeVeque). 


\section{Introduction}

In many physical applications, geometric considerations require the use of numerical grids that are not Cartesian. If the solution domain is a flat manifold but contains complicated internal or external boundaries, it is often possible to introduce a curvilinear grid that conforms to the boundaries [41]. A fundementally different situation arises when the solution domain is a curved manifold, such as the surface of a sphere of radius $r$ embedded in $\mathbb{R}^{3}$. In this situation, the curvature of the manifold modifies the underlying dynamics.

In this paper we are specifically interested in the solution of hyperbolic partial differential equations on curved manifolds. A few examples of problems in which one must solve hyperbolic PDEs on a curved manifold include the propagation of sound waves on curved surfaces (e.g., [42]), shallow water dynamics on the surface of planet as a simplified model of the atmosphere (e.g., $[4,19,33])$, and the propagation of magneto-gravity waves in the solar tachocline (e.g., $[12,38,39])$. Another example arises in the study of relativistic flow of matter in the presence of compact objects such as neutron stars and black holes. In this example, the equations of relativisitc gas dynamics must be solved on a space-time manifold that is curved due to the gravitational force of the object (e.g., [11,29,32]).

For curvilinear grids, a standard numerical approach is to update the hyperbolic system in Cartesian form, or sometimes referred to as strong conservationform, in order to avoid the introduction of source terms [43]. However, this strategy does not work for general curved manifolds. Philosophically speaking, there are two approaches one can take in order to solve PDEs on a curved manifold $\mathcal{M} \subset \mathbb{R}^{n}$. The first approach is not to directly solve the equations on the manifold, but instead to solve the equations in Cartesian form in $\mathbb{R}^{n}$ with the help of a Lagrange multiplier to force the solution to remain on $\mathcal{M} \subset \mathbb{R}^{n}$ (e.g., $[5,40]$ ). The advantage is that all of the geometry is hidden in a relatively simple source term; the disadvantage is that one must solve on a higher-dimensional domain. The alternative to this strategy is to solve directly on the manifold. This removes the extra space dimension, but introduces geometric source terms and flux functions that explicitly vary in space. This strategy has been widely used in numerical simulations of shallow water on the surface of a sphere (e.g., $[4,19,33])$ as well as relativistic hydrodynamics

(e.g., $[11,29,32])$, In this work we will pursue the approach of directly solving on the curved manifold.

In particular, we present in this work a numerical scheme that is a generalization of the wave propagation method [23,24,27]. The wave propagation method is a high-resolution finite volume method for solving hyperbolic systems. The basic algorithm applies to equations in Cartesian coordinates, although it has 
also been extended to general quadrilateral grids on flat manifolds (see Chapter 23 of [27]). The method is numerically conservative, second order accurate in smooth regions, non-dispersive in regions of large gradients, and shockcapturing. This method has been successfully applied in the past to several applications areas, including gas dynamics $[21]$, acoustics $[9,10]$, elasticity and plasticity $[8,22,26,27]$, combustion and detonation waves $[16,17]$, relativistic hydrodynamics [1], and numerical relativity [3,18].

The philosophy pursued in our modified wave propagation algorithm is described below:

(1) The hyperbolic system is solved in the coordinate basis defined by the numerical grid. Components of vector unknowns are updated in contravariant form. In this form, the equations become a system of balance laws with flux functions that depend explicitly on the spatial coordinates and with geometric source terms that appear in the equations for the vector unknowns.

(2) For smooth flows the algorithm produces a second order accurate approximation in space and time: $\mathcal{O}\left(|\Delta \vec{x}|^{2}, \Delta t^{2}\right)$.

(3) The algorithm is shock-capturing.

(4) The explicit spatial variation present in the flux functions is handled through the use of an approximate Riemann solver that is based on decomposing flux differences.

(5) With this approach no operator splitting is required. The geometric source term is discretized through the use of parallel transport.

(6) All necessary Riemann problems are solved in a locally valid orthonormal basis. This removes all geometric terms and reduces the original coordinate Riemann problem to a Cartesian Riemann problem.

The above philosophy results in a wave propagation algorithm that requires only the knowledge of the underlying metric and how to solve orthonormal Riemann problems. The interaction of the geometry with the hyperbolic wave propagation is handled internally by the algorithm.

For simplicity, we focus specifically on manifolds that can be described by two independent coordinates. The numerical method presented in this paper extends in a straightforward manner to manifolds described by three independent coordinates. The corrections introduced by Langseth and LeVeque [21] can be used to approximate the double transverse terms that arise in three dimensions. Key concepts such as parallel transport (see Section 5.1) and orthonormalization (see Section 5.3) also naturally extend to higher dimensions. Some discussion of this can be found in [1]. 


\section{Notation and some concepts from differential geometry}

We first explain some of the notation used in this paper and introduce some basic concepts from differential geometry. A summary of the notation that will be used throughout this paper can be found in Table 1. Unless otherwise noted, summation is implied whenever the same index appears as both a subscript and a superscript. For example,

$$
\Gamma_{n k}^{m} T^{k n}=\sum_{n} \sum_{k} \Gamma_{n k}^{m} T^{k n}
$$

\begin{tabular}{|c||c|}
\hline Symbol & Description \\
\hline \hline$\vec{x}, x^{k}$ & vector in physical space, $k^{\text {th }}$ component \\
\hline$\stackrel{\leftrightarrow}{T}, T^{m n}$ & tensor in physical space, $m n^{\text {th }}$ component \\
\hline $\boldsymbol{q}, q^{k}$ & vector in state space, $k^{\text {th }}$ component \\
\hline $\overrightarrow{\boldsymbol{f}}, \boldsymbol{f}^{k}$ & tensor: rows in physical, columns in state, $k^{\text {th }}$ column \\
\hline$\overleftrightarrow{h}, h_{m n}, h^{m n}$ & metric tensor, $m n^{\text {th }}$ component, $m n^{\text {th }}$ component of inverse \\
\hline$\sqrt{h}$ & square root of the determinant of the metric \\
\hline$\vec{e}_{k}, \tilde{e}^{n}$ & $k^{\text {th }}$ coordinate basis vector, $n^{\text {th }}$ coordinate co-basis vector \\
\hline$\Gamma_{m n}^{k}$ & $k m n^{\text {th }}$ Christoffel symbol \\
\hline
\end{tabular}

Table 1

Summary of notation.

\subsection{Curved manifolds}

Differential geometry describes the geometric structure of a curved differentiable manifold, $\mathcal{M}$. For example, a manifold $\mathcal{M}$ may represent the nearly spherical surface of a planet, a curved spacetime in relativity theory, or the surface of an elastic membrane. We develop an intuitive notion of a manifold by the following heuristic definition:

A manifold is a set of points that looks locally Euclidean in that this set can be entirely covered by a collection of local coordinate mappings.

In this work we consider a two-dimensional curved manifold that is embedded in $\mathbb{R}^{3}$. Let the coordinates $\left(x^{1}, x^{2}\right)$ be the coordinates on the manifold $\mathcal{M}$. This coordinate system can be related to the standard Cartesian coordinate 
system, $(x, y, z)$, through the transformations

$$
\begin{aligned}
& x=X\left(x^{1}, x^{2}\right) \\
& y=Y\left(x^{1}, x^{2}\right) \\
& z=Z\left(x^{1}, x^{2}\right) .
\end{aligned}
$$

A vector, $\left(\mu^{1}, \mu^{2}\right)$, in contravariant form on the manifold $\mathcal{M}$ can be transformed to a vector, $\left(\mu^{x}, \mu^{y}, \mu^{z}\right)$, in Cartesian space through the Jacobian $J$ in the following way:

$$
\left[\begin{array}{l}
\mu^{x} \\
\mu^{y} \\
\mu^{z}
\end{array}\right]=J\left[\begin{array}{l}
\mu^{1} \\
\mu^{2}
\end{array}\right]=\left[\begin{array}{ll}
\frac{\partial X}{\partial x^{1}} & \frac{\partial X}{\partial x^{2}} \\
\frac{\partial Y}{\partial x^{1}} & \frac{\partial Y}{\partial x^{2}} \\
\frac{\partial Z}{\partial x^{1}} & \frac{\partial Z}{\partial x^{2}}
\end{array}\right]\left[\begin{array}{l}
\mu^{1} \\
\mu^{2}
\end{array}\right]
$$

Therefore, the coordinate transformations directly give us a natural basis in which to represent vectors on $\mathcal{M}$. We will refer to such a basis as a coordinate basis.

\subsection{The metric tensor}

The metric tensor, $\overleftrightarrow{h}$, is a symmetric tensor that provides a measure of length on $\mathcal{M}$. The metric relates true distances as measured in $\mathbb{R}^{3}$ to the coordinate distances measured in the coordinate system of the manifold. In particular, the line element $d s^{2}=(d x)^{2}+(d y)^{2}+(d z)^{2}$ in $\mathbb{R}^{3}$ is related to $d x^{1}$ and $d x^{2}$ through

$$
d s^{2}=h_{m n} d x^{m} d x^{n} .
$$

The distance along a curve $C(\lambda)$ parameterized by $\lambda$ from $C(a)$ to $C(b)$ is given by

$$
L=\int_{a}^{b}\left|h_{m n}\left(\frac{d x^{m}}{d \lambda}\right)\left(\frac{d x^{n}}{d \lambda}\right)\right|^{1 / 2} d \lambda .
$$

The surface area of $\Omega \subseteq \mathcal{M}$ can be evaluated by computing the following integral:

$$
\text { Surface Area of } \Omega=\int_{\Omega\left(x^{1}, x^{2}\right)} \sqrt{h} d x^{1} d x^{2} \text {, }
$$

where $\sqrt{h}$ is the square root of the determinant of the metric tensor.

Using the Jacobian matrix discussed above, the metric can be constructed as follows:

$$
\overleftrightarrow{h}=J^{t} J
$$


Furthermore, if the metric tensor is non-singular we denote the inverse of the metric tensor by

$$
h^{m n} \equiv\left[\stackrel{\leftrightarrow}{h}^{-1}\right]_{m n}
$$

This implies that

$$
h_{m k} h^{k n}=\delta_{m}^{n}
$$

where $\delta_{m}^{n}$ is the Kroenecker delta.

\subsection{Christoffel symbols}

The final concept from differential geometry that we need to introduce is the Christoffel symbol. The components of the basis $\left(\vec{e}_{k}\right)$, co-basis $\left(\tilde{e}^{n}\right)$, and the metric tensor will in general be functions of $x^{1}$ and $x^{2}$. It is therefore necessary to modify the notion of a derivative.

For example, the gradient of vector $\vec{U}$ with contravariant components $U^{k}$ is given by the following [31]:

$$
\vec{\nabla} \vec{U}=\left[\frac{\partial}{\partial x^{n}} U^{k}+\Gamma_{m n}^{k} U^{m}\right] \tilde{e}^{n} \otimes \vec{e}_{k}
$$

where $\otimes$ is the outer product. In this expression, $\partial U^{k} / \partial x^{n}$ is the usual term one would expect when computing $\vec{\nabla} \vec{U}$ in a Cartesian basis. The additional term, $\Gamma_{m n}^{k} U^{m}$, appears because the underlying basis is spatially varying.

Similarly, the gradient of a tensor $\stackrel{\leftrightarrow}{T}$ can be written

$$
\vec{\nabla} \stackrel{\leftrightarrow}{T}=\left[\frac{\partial}{\partial x^{l}} T^{k m}+\Gamma_{n l}^{k} T^{n m}+\Gamma_{n l}^{m} T^{k n}\right] \tilde{e}^{l} \otimes \vec{e}_{k} \otimes \vec{e}_{m}
$$

In this case, two additional terms, $\Gamma_{n l}^{k} T^{n m}$ and $\Gamma_{n l}^{m} T^{k n}$, appear because the underlying basis is spatially varying.

The components of the tensor $\Gamma_{m n}^{k}$ are referred to as the Christoffel symbols or as connection coefficients. They involve spatial derivatives of the metric tensor $\stackrel{\leftrightarrow}{h}$. In particular, in a coordinate basis they can be written as follows (see Misner, Thorne, and Wheeler [31]):

$$
\Gamma_{m n}^{k} \equiv \frac{1}{2} h^{\alpha k}\left(\frac{\partial}{\partial x^{n}} h_{\alpha m}+\frac{\partial}{\partial x^{m}} h_{\alpha n}-\frac{\partial}{\partial x^{\alpha}} h_{m n}\right) .
$$

As will be seen in the next section, the Christoffel symbols play an important role in wave propagation on curved manifolds. 


\section{Conservation laws on curved manifolds}

Consider the flow of a substance with $M$ state variables, $\boldsymbol{q}(\vec{x}, t) \in \mathbb{R}^{M}$. In the absence of any sources or sinks, the time rate of change of the integral of each state variable over the volume $\mathcal{V}$ is only dependent on the flux of that variable through the boundary, $\partial \mathcal{V}$. Mathematically, this is expressed with the following integral conservation law

$$
\frac{\partial}{\partial t} \int_{\mathcal{V}} \boldsymbol{q}(t, \vec{x}) d \mathcal{V}+\int_{d \mathcal{V}} \overrightarrow{\boldsymbol{f}}(\boldsymbol{q}) \cdot \vec{n} d s=0
$$

where $\overrightarrow{\boldsymbol{f}}(\boldsymbol{q}) \in \mathbb{R}^{M \times 2}$ is the flux function, $\vec{n}$ is the outward pointing unit normal vector to $d \mathcal{V}$, and $s$ is the arclength parameterization of $d \mathcal{V}$.

The differential form of (14) is written

$$
\frac{\partial}{\partial t} \boldsymbol{q}+\vec{\nabla} \cdot \overrightarrow{\boldsymbol{f}}(\boldsymbol{q})=0
$$

and is well known to a diverse community of computational fluid dynamicists. It is also well known that the integral conservation law may exhibit discontinuous solutions that satisfy a weak form of (15) together with an entropy condition imposed by the underlying physics of the system at hand.

In order to solve (15), it is necessary to express the conservation law in some basis. In flat space, equation (15) is often written in a Cartesian basis:

$$
\frac{\partial}{\partial t} \boldsymbol{q}+\frac{\partial}{\partial x} \boldsymbol{f}^{x}(\boldsymbol{q})+\frac{\partial}{\partial y} \boldsymbol{f}^{y}(\boldsymbol{q})=0
$$

Note that $\boldsymbol{f}^{x}(\boldsymbol{q})$ and $\boldsymbol{f}^{y}(\boldsymbol{q})$ do not depend explicitly on space and that no geometric source terms are evident. In many interesting applications, however, we are required to solve (15) on a smooth manifold $\mathcal{M}$ covered by a set of non-Cartesian basis vectors. In general, the coordinate basis representation of (15) takes the form of a balance law:

$$
\frac{\partial}{\partial t} \boldsymbol{q}+\frac{\partial}{\partial x^{1}} \boldsymbol{f}^{1}(\boldsymbol{q}, \vec{x})+\frac{\partial}{\partial x^{2}} \boldsymbol{f}^{2}(\boldsymbol{q}, \vec{x})=\boldsymbol{\psi}_{c}(\boldsymbol{q}, \vec{x})
$$

where the flux has now gained an explicit dependence on the spatial coordinates and a geometrically induced source term has appeared.

To further understand equation (17) let us consider a conservation law in which $\boldsymbol{q}$ is comprised of a scalar quantity $\rho(\vec{x}, t)$ (e.g., density) and a vector 
quantity $\vec{\mu}(\vec{x}, t)$ (e.g., momentum):

$$
\boldsymbol{q}(\vec{x}, t)=\left[\begin{array}{l}
\rho \\
\vec{\mu}
\end{array}\right] .
$$

Let the corresponding flux function be

$$
\overrightarrow{\boldsymbol{f}}(\boldsymbol{q}, \vec{x})=\left[\begin{array}{l}
\vec{U} \\
\stackrel{T}{T}
\end{array}\right]
$$

The divergence of a vector $\vec{U}$ can be computed by taking the trace of $\vec{\nabla} \vec{U}$. $\vec{\nabla} \vec{U}$ is given by (11) and the trace of this expression is obtained if we contract by letting $n=k$ (which implies summation on $k$ ):

$$
\vec{\nabla} \cdot \vec{U}=\frac{\partial}{\partial x^{k}} U^{k}+\Gamma_{m k}^{k} U^{m}=\frac{1}{\sqrt{h}} \frac{\partial}{\partial x^{k}}\left(\sqrt{h} U^{k}\right)
$$

where we have made use of the fact that

$$
\Gamma_{m k}^{k}=\frac{1}{\sqrt{h}} \frac{\partial}{\partial x^{m}}(\sqrt{h})
$$

Similarly, the divergence of a tensor can be obtained if we contract expression (12) by letting $l=k$ :

$$
\begin{aligned}
\vec{\nabla} \cdot \stackrel{\leftrightarrow}{T} & =\left(\frac{\partial}{\partial x^{k}} T^{k m}+\Gamma_{n k}^{k} T^{n m}+\Gamma_{n k}^{m} T^{k n}\right) \vec{e}_{m} \\
& =\left(\frac{1}{\sqrt{h}} \frac{\partial}{\partial x^{k}}\left(\sqrt{h} T^{k m}\right)+\Gamma_{n k}^{m} T^{k n}\right) \vec{e}_{m}
\end{aligned}
$$

Using results (20) and (22), we can rewrite equation (15) in coordinate form:

$$
\begin{aligned}
\frac{\partial}{\partial t} \rho+\frac{1}{\sqrt{h}} \frac{\partial}{\partial x^{k}}\left(\sqrt{h} U^{k}\right) & =0 \\
\frac{\partial}{\partial t} \mu^{m}+\frac{1}{\sqrt{h}} \frac{\partial}{\partial x^{k}}\left(\sqrt{h} T^{k m}\right) & =-\Gamma_{n k}^{m} T^{k n} .
\end{aligned}
$$

The focus of this paper is to develop an accurate numerical method for the solution of equations (23) and (24). Note that both the scalar equation (23) and the vector equation (24) have flux functions that explicitly vary in space. Furthermore, the vector equation (24) has an additional geometric source term that describes transfer between $\mu^{1}(\vec{x}, t)$ and $\mu^{2}(\vec{x}, t)$ due to spatial variation in the coordinate basis. 


\section{The wave propagation method in Cartesian coordinates}

In this section we introduce the wave propagation method of LeVeque [24]. In particular, we focus on aspects of the method that are relevant for the wave propagation method on curved manifolds.

\subsection{Cartesian finite volume methods}

Let us fist consider Cartesian conservation laws of the form (16). We construct a Cartesian grid with grid spacing $\Delta x$ and $\Delta y$ and let

$$
\begin{aligned}
& x_{i}=x_{\ell}+\left(i-\frac{1}{2}\right) \Delta x \\
& y_{j}=y_{\ell}+\left(j-\frac{1}{2}\right) \Delta y,
\end{aligned}
$$

where $\left(x_{\ell}, y_{\ell}\right)$ is the lower-left corner of the rectangular computational domain. In each grid cell centered at $\left(x_{i}, y_{j}\right)$ and at each time $t^{n}$, a finite volume method will produce an approximation to the average of $\boldsymbol{q}(\vec{x}, t)$ :

$$
\boldsymbol{Q}_{i j}^{n} \approx \frac{1}{\Delta x \Delta y} \int_{y_{j-1 / 2}}^{y_{j+1 / 2}} \int_{x_{i-1 / 2}}^{x_{i+1 / 2}} \boldsymbol{q}\left(\xi, \eta, t^{n}\right) d \xi d \eta
$$

If $\Delta t \equiv t^{n+1}-t^{n}$, then the time averaged flux of $\boldsymbol{q}$ from $t=t^{n}$ to $t=t^{n+1}$ across the cell interface located at $x_{i-1 / 2}$ and the cell interface located at $y_{j-1 / 2}$ can be written as

$$
\begin{aligned}
& \boldsymbol{F}_{i-1 / 2, j}^{1} \approx \frac{1}{\Delta t} \int_{t^{n}}^{t^{n+1}} \boldsymbol{f}^{x}\left(\boldsymbol{q}\left(\vec{x}_{i-1 / 2, j}, \tau\right)\right) d \tau \\
& \boldsymbol{F}_{i, j-1 / 2}^{2} \approx \frac{1}{\Delta t} \int_{t^{n}}^{t^{n+1}} \boldsymbol{f}^{y}\left(\boldsymbol{q}\left(\vec{x}_{i, j-1 / 2}, \tau\right)\right) d \tau,
\end{aligned}
$$

respectively. Conservation now tells us that $\boldsymbol{Q}_{i j}^{n+1}$ must be equal to $\boldsymbol{Q}_{i j}^{n}$ minus the flux of $\boldsymbol{Q}$ out of the grid cell centered at $\left(x_{i}, y_{j}\right)$ :

$$
\boldsymbol{Q}_{i j}^{n+1}=\boldsymbol{Q}_{i j}^{n}-\frac{\Delta t}{\Delta x}\left(\boldsymbol{F}_{i+1 / 2, j}^{1}-\boldsymbol{F}_{i-1 / 2, j}^{1}\right)-\frac{\Delta t}{\Delta y}\left(\boldsymbol{F}_{i, j+1 / 2}^{2}-\boldsymbol{F}_{i, j-1 / 2}^{2}\right)
$$

All Cartesian finite volume methods can be written in the form (30). A full numerical scheme is obtained by choosing a specific strategy for constructing the numerical fluxes $\boldsymbol{F}^{1}$ and $\boldsymbol{F}^{2}$. Independent of how these fluxes are computed, however, update (30) guarantees numerical conservation [27]. 


\subsection{The standard wave propagation method}

In the wave propagation method, update (30) is rewritten in a slighly different form:

$$
\begin{aligned}
\boldsymbol{Q}_{i j}^{n+1}=\boldsymbol{Q}_{i j}^{n} & -\frac{\Delta t}{\Delta x}\left[\mathcal{A}_{1}^{-} \Delta \boldsymbol{Q}_{i+1 / 2, j}+\mathcal{A}_{1}^{+} \Delta \boldsymbol{Q}_{i-1 / 2, j}\right] \\
& -\frac{\Delta t}{\Delta y}\left[\mathcal{A}_{2}^{-} \Delta \boldsymbol{Q}_{i, j+1 / 2}+\mathcal{A}_{2}^{+} \Delta \boldsymbol{Q}_{i, j-1 / 2}\right] \\
& -\frac{\Delta t}{\Delta x}\left[\tilde{\boldsymbol{F}}_{i+1 / 2, j}^{1}-\tilde{\boldsymbol{F}}_{i-1 / 2, j}^{1}\right]-\frac{\Delta t}{\Delta y}\left[\tilde{\boldsymbol{F}}_{i, j+1 / 2}^{2}-\tilde{\boldsymbol{F}}_{i, j-1 / 2}^{2}\right],
\end{aligned}
$$

where $\mathcal{A}^{-} \Delta \boldsymbol{Q}$ and $\mathcal{A}^{+} \Delta \boldsymbol{Q}$ are fluctuations. The wave propagation method is constructed so that the fluctuations contain corrections needed for a first order Godunov update and the numerical fluxes $\tilde{\boldsymbol{F}}^{1}$ and $\tilde{\boldsymbol{F}}^{2}$ contain higher order corrections [24,27].

In the standard wave propagation method the fluctuations are constructed by solving locally linearized Riemann problems. At each cell interface $\left(x_{i-1 / 2}, y_{j}\right)$ we have to solve a Riemann problem of the form

$$
\begin{gathered}
\frac{\partial}{\partial t} \boldsymbol{q}+\tilde{\boldsymbol{A}}_{i-1 / 2, j} \frac{\partial}{\partial x} \boldsymbol{q}=0 \\
\boldsymbol{q}(x, 0)= \begin{cases}\boldsymbol{Q}_{i-1, j} & \text { if } x<x_{i-1 / 2} \\
\boldsymbol{Q}_{i j} & \text { if } x>x_{i-1 / 2}\end{cases}
\end{gathered}
$$

where

$$
\tilde{\boldsymbol{A}}_{i-1 / 2, j} \approx \frac{\partial \boldsymbol{f}^{x}}{\partial \boldsymbol{q}}\left(\boldsymbol{q}\left(\vec{x}_{i-1 / 2, j}, t\right), \vec{x}_{i-1 / 2, j}\right)
$$

is an approximate flux Jacobian that is considered to be constant in the Riemann problem. We denote the eigenvalues, right eigenvectors, and left eigenvectors of $\tilde{\boldsymbol{A}}_{i-1 / 2, j}$ by $s_{i-1 / 2, j}^{p}, \boldsymbol{r}_{i-1 / 2, j}^{p}$, and $\boldsymbol{\ell}_{i-1 / 2, j}^{p}$.

In order to solve Riemann problem (32)-(33), the difference between $\boldsymbol{Q}_{i j}$ and $\boldsymbol{Q}_{i-1, j}$ is decomposed along the eigenvectors of $\tilde{\boldsymbol{A}}_{i-1 / 2, j}$. Based on these eigenvectors and eigenvalues, the fluctuations $\mathcal{A}_{1}^{-} \Delta \boldsymbol{Q}_{i-1 / 2, j}$ and $\mathcal{A}_{1}^{+} \Delta \boldsymbol{Q}_{i-1 / 2, j}$ in (31) are assembled by summing all the components of the flux that are left- 
and right-going, respectively. Mathematically, we write

$$
\begin{aligned}
\boldsymbol{Q}_{i j}-\boldsymbol{Q}_{i-1, j} & =\sum_{p=1}^{M} \boldsymbol{W}_{i-1 / 2, j}^{p} \\
\mathcal{W}_{i-1 / 2, j}^{p} & =\left[\boldsymbol{\ell}_{i-1 / 2, j}^{p} \cdot\left(\boldsymbol{Q}_{i j}-\boldsymbol{Q}_{i-1, j}\right)\right] \boldsymbol{r}_{i-1 / 2, j}^{p} \\
\mathcal{A}_{1}^{+} \Delta \boldsymbol{Q}_{i-1 / 2, j} & =\sum_{p: s_{i-1 / 2, j}^{p}>0}^{p} s_{i-1 / 2, j}^{p} \mathcal{W}_{i-1 / 2, j}^{p} \\
\mathcal{A}_{1}^{-} \Delta \boldsymbol{Q}_{i-1 / 2, j} & =\sum_{p: s_{i-1 / 2, j}^{p}<0}^{p} s_{i-1 / 2, j}^{p} \mathcal{W}_{i-1 / 2, j}^{p},
\end{aligned}
$$

where we refer to $\mathcal{W}_{i-1 / 2}^{p}$ as a wave and $s_{i-1 / 2, j}^{p}$ as a wave speed. To obtain the fluctuations $\mathcal{A}_{2}^{-} \Delta \boldsymbol{Q}_{i, j-1 / 2}$ and $\mathcal{A}_{2}^{+} \Delta \boldsymbol{Q}_{i, j-1 / 2}$, an analogous process to that of (35) - (38) must be applied in the 2-direction.

It is well known that linearized Riemann solvers such as the one presented here can produce entropy violating solutions for transonic rarefactions $[7,27]$. A transonic rarefaction occurs when the solution to the Riemann problem contains a rarefaction that is part left-going and part right-going with respect to the reference frame fixed at $\left(x_{i-1 / 2}, y_{j}\right)$. In this case, the linearized Riemann problem will represent this rarefaction as a jump discontinuity which is either left or right traveling. This problem can be corrected by redefining fluctuations (37) and (38) so that locally we obtain a more diffusive numerical update that gives the correct entropy satisfying solution. In particular, we utilize an entropy fix similar to the one devised by Harten and Hyman [13]. We refer the reader to Chapter 15 of LeVeque [27] for more discussion on the implementation of this entropy fix in the wave propagation method framework.

Without additional corrections, the wave propagation method so far is only $\mathcal{O}(|\Delta \vec{x}|, \Delta t)$ accurate and in 2-dimensions only stable up to a Courant number of $1 / 2[23,24,27]$. To overcome these difficulties, higher order correction fluxes are introduced. To eliminate the numerical diffusion introduced by the first order method, the high-resolution correction is constructed:

$$
\tilde{\boldsymbol{F}}_{i-1 / 2, j}^{1}=\frac{1}{2} \sum_{p=1}^{M}\left|s_{i-1 / 2, j}^{p}\right|\left(1-\frac{\Delta t}{\Delta x}\left|s_{i-1 / 2, j}^{p}\right|\right) \boldsymbol{W}_{i-1 / 2}^{p} \phi_{i-1 / 2, j}
$$

where $\phi_{i-1 / 2}$ is a wave limiter. When $\phi \equiv 1$, the wave propagation method reduces to the classical Lax-Wendroff method for linear problems. In general, however, the wave limiter allows the method to switch between a second order method in smooth regions of the solution and a first order method near discontinuities. Finally, in order to make this method stable up to Courant number 1 without dimensional splitting, transverse corrections based on solving transverse Riemann problems are added to the numerical fluxes. For more details on these corrections, we refer the reader to $[23,24,27]$. 


\subsection{Capacity form differencing}

One generalization of the above wave propagation method that will be required when solving conservation laws (23) and (24) on curved manifolds is the inclusion of a capacity function [24,27]. In (23) and (24), the square root

of the determinant of the metric, $\sqrt{h}$, acts as a capacity function. In Cartesian coordinates, a conservation law with a capacity function $\kappa(\vec{x})>0$ can be written as

$$
\frac{\partial}{\partial t}(\kappa \boldsymbol{q})+\frac{\partial}{\partial x} \boldsymbol{f}^{x}(\boldsymbol{q})+\frac{\partial}{\partial y} \boldsymbol{f}^{y}(\boldsymbol{q})=0 .
$$

The cell average of $q$ in the grid cell centered at $\left(x_{i}, y_{j}\right)$ is now given by

$$
\boldsymbol{Q}_{i j}^{n} \approx \frac{1}{\kappa_{i j} \Delta x \Delta y} \int_{y_{j-1 / 2}}^{y_{j+1 / 2}} \int_{x_{i-1 / 2}}^{x_{i+1 / 2}} \kappa(\xi, \eta) \boldsymbol{q}\left(\xi, \eta, t^{n}\right) d \xi d \eta
$$

instead of (27). The normal and transverse Riemann problems remain unaltered by the capacity function. The high-resolution correction fluxes (39), the transverse correction fluxes, and update (31) must all be modified by replacing all the $\Delta t$ 's that appear in these expressions by $\Delta t / \kappa$ evaluated at the appropriate spatial location $[24,27]$. These formulas are given in detail in Section 6 for wave propagation algorithm on curved manifolds.

\subsection{Spatially varying flux functions}

In this and the next section, we briefly summarize two key results from Bale et. al [2]. These results will be important when we go to solve equations (23) and (24) on a curved manifold. Consider a Cartesian conservation law with flux functions that explicitly vary in space:

$$
\frac{\partial}{\partial t} \boldsymbol{q}+\frac{\partial}{\partial x} \boldsymbol{f}^{x}(\boldsymbol{q}, \vec{x})+\frac{\partial}{\partial y} \boldsymbol{f}^{y}(\boldsymbol{q}, \vec{x})=0 .
$$

The method presented in [2] relies on modifying the approximate Riemann problems that are solved at each grid interface to take into account the spatial variation in the flux.

The key difference between the original wave propagation method [24] and the modifications presented in Bale et al. [2] is that in [2] we solve the approximate Riemann problem by decomposing flux differences instead of differences in $\boldsymbol{Q}$. Instead of the Riemann problem (32)-(33), we must now solve, at each cell 
interface $\left(x_{i-1 / 2}, y_{j}\right)$, a generalized Riemann problem of the form:

$$
\begin{gathered}
\frac{\partial}{\partial t} \boldsymbol{q}+\frac{\partial}{\partial x} \boldsymbol{f}^{x}(\boldsymbol{q}, x)=0 \\
\boldsymbol{q}(x, 0)= \begin{cases}\boldsymbol{Q}_{i-1, j} & \text { if } x<x_{i-1 / 2} \\
\boldsymbol{Q}_{i j} & \text { if } x>x_{i-1 / 2}\end{cases} \\
\boldsymbol{f}^{x}(\boldsymbol{q}, x)= \begin{cases}\boldsymbol{f}_{i-1, j}^{x}(\boldsymbol{q}) & \text { if } x<x_{i-1 / 2} \\
\boldsymbol{f}_{i j}^{x}(\boldsymbol{q}) & \text { if } x>x_{i-1 / 2}\end{cases}
\end{gathered}
$$

If the Rankine-Hugoniot conditions are used to test for a standing wave solution $(s=0)$ across the cell interface at $x=x_{i-1 / 2}$ we obtain the following result:

$$
\boldsymbol{f}_{i j}^{x}\left(\boldsymbol{Q}^{+}\right)-\boldsymbol{f}_{i-1, j}^{x}\left(\boldsymbol{Q}^{-}\right)=0 .
$$

Because $\boldsymbol{f}_{i j}^{x}(\boldsymbol{q}) \neq \boldsymbol{f}_{i-1, j}^{x}(\boldsymbol{q})$ it follows from (46) that $\boldsymbol{q}(x, t>0)$ must be discontinous and $\boldsymbol{f}^{x}(x, t>0)$ must be continuous across $x=x_{i-1 / 2}$. Therefore, a wave decomposition based on $\boldsymbol{Q}$ differences would have to correct for the additional jump across $x=x_{i-1 / 2}$. A wave decomposition based on flux differences, on the other hand, requires only the usual $M$ waves and the additional standing wave $Q^{+}-Q^{-}$is automatically generated because (46) is exactly satisfied.

The modified wave decomposition now takes the following form:

$$
\Delta \boldsymbol{F}^{1} \equiv \boldsymbol{f}^{x}\left(\boldsymbol{Q}_{i j}, \vec{x}_{i j}\right)-\boldsymbol{f}^{x}\left(\boldsymbol{Q}_{i-1, j}, \vec{x}_{i-1, j}\right)=\sum_{p=1}^{M} \mathcal{Z}_{i-1 / 2, j}^{p}
$$

where

$$
\mathcal{Z}_{i-1 / 2, j}^{p}=\left[\boldsymbol{\ell}_{i-1 / 2, j}^{p} \cdot \Delta \boldsymbol{F}^{1}\right] \boldsymbol{r}_{i-1 / 2, j}^{p} .
$$

The fluctuations in update (31) are now

$$
\begin{aligned}
\mathcal{A}_{1}^{+} \Delta \boldsymbol{Q}_{i-1 / 2, j} & =\sum_{p: s_{i-1 / 2, j}^{p}>0} \mathcal{Z}_{i-1 / 2, j}^{p}+\frac{1}{2} \mathbb{Z} \\
\mathcal{A}_{1}^{-} \Delta \boldsymbol{Q}_{i-1 / 2, j} & =\sum_{p: s_{i-1 / 2, j}^{p}<0} \mathcal{Z}_{i-1 / 2, j}^{p}+\frac{1}{2} \mathbb{Z},
\end{aligned}
$$

where

$$
\mathbb{Z}_{i-1 / 2, j}=\sum_{p: s_{i-1 / 2, j}^{p}=0} \mathcal{Z}_{i-1 / 2, j}^{p} .
$$

In Rossmanith [35] it is shown that if $s_{i-1 / 2, j}^{p}=0$ for some $1 \leq p \leq m$ then $\mathbb{Z}_{i-1 / 2, j} \equiv 0$ if the approximate flux Jacobian satisfies

$$
\tilde{\boldsymbol{A}}_{i-1 / 2, j}\left(\boldsymbol{Q}_{i j}-\boldsymbol{Q}_{i-1, j}\right)=\boldsymbol{f}^{x}\left(\boldsymbol{Q}_{i j}, \vec{x}_{i j}\right)-\boldsymbol{f}^{x}\left(\boldsymbol{Q}_{i-1, j}, \vec{x}_{i-1, j}\right)
$$


In general, however, $\mathbb{Z}_{i-1 / 2, j} \neq 0$ if $s_{i-1 / 2, j}^{p}=0$ for some $1 \leq p \leq m$. Independent of the approximate flux Jacobian, however, the above wave decomposition guarantees that update (31) is numerically conservative. This is because

$$
\mathcal{A}_{1}^{+} \Delta \boldsymbol{Q}_{i-1 / 2, j}+\mathcal{A}_{1}^{-} \Delta \boldsymbol{Q}_{i-1 / 2, j}=\boldsymbol{f}^{x}\left(\boldsymbol{Q}_{i j}, \vec{x}_{i j}\right)-\boldsymbol{f}^{x}\left(\boldsymbol{Q}_{i-1, j}, \vec{x}_{i-1, j}\right)
$$

by construction (47).

The linearized Riemann solver based on (47), (48), (49), and (50) again fails to produce the correct entropy solution in the case of a transonic rarefaction. A transonic rarefaction will occur in the $p^{\text {th }}$ wave family if

$$
\lambda_{l}^{p}<0 \quad \text { and } \quad \lambda_{r}^{p}>0
$$

where $\lambda_{l, r}^{p}$ represents the $p^{\text {th }}$ eigenvalue of the flux Jacobian evaluated at $Q_{i-1, j}$ and $Q_{i j}$, respectively. If a transonic rarefaction occurs, we apply a modified version of the Harten and Hyman [13] approach. In particular we define the quantity

$$
\theta^{p}=\left(\frac{s^{p}-\lambda_{r}^{p}}{\lambda_{l}^{p}-\lambda_{r}^{p}}\right)\left(\frac{\lambda_{l}^{p}}{s^{p}}\right)
$$

and place the wave, $\mathcal{Z}^{p}$, partly into the left fluctuation and partly into the right fluctuation:

$$
\begin{aligned}
& \mathcal{A}_{1}^{-} \Delta \boldsymbol{Q}=\mathcal{A}_{1}^{-} \Delta \boldsymbol{Q}+\theta^{p} \mathcal{Z}^{p} \\
& \mathcal{A}_{1}^{+} \Delta \boldsymbol{Q}=\mathcal{A}_{1}^{+} \Delta \boldsymbol{Q}+\left(1-\theta^{p}\right) \mathcal{Z}^{p} .
\end{aligned}
$$

Note that this maintains numerical conservation.

Next, the high-resolution correction flux (39) is replaced by

$$
\tilde{\boldsymbol{F}}_{i-1 / 2, j}^{1}=\frac{1}{2} \sum_{p=1}^{M} \operatorname{sign}\left(s_{i-1 / 2, j}^{p}\right)\left(1-\frac{\Delta t}{\Delta x}\left|s_{i-1 / 2, j}^{p}\right|\right) \tilde{\mathcal{Z}}_{i-1 / 2, j}^{p},
$$

where

$$
\tilde{\mathcal{Z}}_{i-1 / 2, j}^{p}=\mathcal{Z}_{i-1 / 2, j}^{p} \phi_{i-1 / 2, j} .
$$

Finally, the general form of the transverse corrections remains unaltered [2].

Bale et al. [2] verify through numerical experiment that the above modifications produce a numerical method that is $\mathcal{O}\left(|\Delta \vec{x}|^{2}, \Delta t^{2}\right)$ accurate for smooth solutions, captures shocks well, and is stable up to Courant number 1. 


\subsection{Source terms}

We present in this section a generalization of the wave propagation method for balance laws of the form:

$$
\frac{\partial}{\partial t} \boldsymbol{q}+\frac{\partial}{\partial x} \boldsymbol{f}^{x}(\boldsymbol{q}, \vec{x})+\frac{\partial}{\partial y} \boldsymbol{f}^{y}(\boldsymbol{q}, \vec{x})=\boldsymbol{\psi}(\boldsymbol{q}, \vec{x})
$$

Results discussed in this section will later be used for obtaining solutions to evolution equation (24) for vector quantities on curved manifolds (see Section $5.2)$.

The approach we describe in this section for handling source terms was first discussed in the context of the wave propagation method in LeVeque and Pelanti [28] and then further developed in Bale et al. [2]. This method completely avoids the need for operator splitting and achieves an accurate numerical solution to (60) through minor modifications of the wave decomposition (47). Because operator splitting is avoided in this approach, steady-states can be more accurately maintained if $\boldsymbol{\psi}^{1}$ and $\boldsymbol{\psi}^{2}$ are chosen appropriately. See [2] and [35] for specific examples.

In order to incorporate the source term into the one-dimensional wave decomposition (47), we must first separate the source term into two distinct pieces:

$$
\boldsymbol{\psi}(\boldsymbol{q}, \vec{x})=\boldsymbol{\psi}^{1}(\boldsymbol{q}, \vec{x})+\boldsymbol{\psi}^{2}(\boldsymbol{q}, \vec{x}) .
$$

It can be shown through simple Taylor series arguments that the order of accuracy of the wave propagation method is unaffected by the specific choice of $\boldsymbol{\psi}^{1}$ and $\boldsymbol{\psi}^{2}$ [35]. The choice only becomes important when the solution is in or near a steady state. If the numerical method is to accurately maintain a discrete form of the steady state, one needs to choose the $\boldsymbol{\psi}^{1}$ and $\boldsymbol{\psi}^{2}$ so that they satisfy the following relationships in the steady state:

$$
\begin{aligned}
\frac{\partial}{\partial x} \boldsymbol{f}^{x}(\boldsymbol{q}, \vec{x}) & =\boldsymbol{\psi}^{1}(\boldsymbol{q}, \vec{x}) \\
\frac{\partial}{\partial y} \boldsymbol{f}^{y}(\boldsymbol{q}, \vec{x}) & =\boldsymbol{\psi}^{2}(\boldsymbol{q}, \vec{x})
\end{aligned}
$$

In some applications, a natural choice for $\boldsymbol{\psi}^{1}$ and $\boldsymbol{\psi}^{2}$ can easily be found. This is true for example in the shallow water equations with bottom topography [25]. In Section 5.2 we will show that a natural choice also exists for conservation laws on curved manifolds.

Bale et al. [2] show that the source term can be incorporated into the wave 
decomposition if we replace (47) by

$$
\Delta \boldsymbol{F}^{1} \equiv \boldsymbol{f}^{x}\left(\boldsymbol{Q}_{i j}, \vec{x}_{i j}\right)-\boldsymbol{f}^{x}\left(\boldsymbol{Q}_{i-1, j}, \vec{x}_{i-1}, j\right)-\Delta x \boldsymbol{\psi}_{i-1 / 2, j}^{1}=\sum_{p=1}^{M} \mathcal{Z}_{i-1 / 2, j}^{p},
$$

where

$$
\boldsymbol{\psi}_{i-1 / 2, j}^{1} \approx \boldsymbol{\psi}\left(\boldsymbol{q}\left(\vec{x}_{i-1 / 2, j}, t\right), \vec{x}_{i-1 / 2, j}\right) .
$$

The rest of the wave decomposition method remains unaltered including the construction of the high-resolution fluxes (58) and the transverse corrections. As is shown in [2], the following corrections must be added to update (31) in order to achieve second order accuracy for smooth solutions:

$$
\boldsymbol{Q}_{i j}^{n+1}=\cdots-\frac{1}{2} \frac{\Delta t^{2}}{\Delta x} \boldsymbol{S}_{i j}^{1}-\frac{1}{2} \frac{\Delta t^{2}}{\Delta y} \boldsymbol{S}_{i j}^{2}
$$

where

$$
\begin{aligned}
& \boldsymbol{S}_{i j}^{1}=\frac{1}{2}\left[\frac{\partial \boldsymbol{\psi}}{\partial \boldsymbol{q}}\right]_{i j} \sum_{p=1}^{M}\left[\tilde{\mathcal{Z}}_{i-1 / 2, j}^{p}+\tilde{\mathcal{Z}}_{i+1 / 2, j}^{p}\right] \\
& \boldsymbol{S}_{i j}^{2}=\frac{1}{2}\left[\frac{\partial \boldsymbol{\psi}}{\partial \boldsymbol{q}}\right]_{i j} \sum_{p=1}^{M}\left[\tilde{\mathcal{Z}}_{i, j-1 / 2}^{p}+\tilde{\mathcal{Z}}_{i, j+1 / 2}^{p}\right] .
\end{aligned}
$$

Corrections (67) and (68) are required for $\mathcal{O}\left(\Delta \vec{x}^{2}, \Delta t^{2}\right)$ accuracy because what is not taken into account in (64) is the time variation of the source term. In fact, we are assuming here that the source term is of the form $\boldsymbol{\psi}(\boldsymbol{q}, \vec{x})$; and therefore, the source term varies in time only through $\boldsymbol{q}(\vec{x}, t)$. If the source term were explicitly dependent on time,

$$
\boldsymbol{\psi}=\boldsymbol{\psi}(\boldsymbol{q}, \vec{x}, t)
$$

then another correction would have to be added to (66) [35]:

$$
\boldsymbol{Q}_{i j}^{n+1}=\cdots-\frac{1}{2} \frac{\Delta t^{2}}{\Delta x} \boldsymbol{S}_{i j}^{1}-\frac{1}{2} \frac{\Delta t^{2}}{\Delta y} \boldsymbol{S}_{i j}^{2}-\frac{1}{2} \Delta t^{2} \frac{\partial \boldsymbol{\psi}}{\partial t} .
$$

\section{The wave propagation method on curved manifolds}

In this section, we develop a method for solving balance laws of the form (23) and (24) on a general curved manifold. Although one could directly apply the algorithm developed in Bale et al. [2] to equations (23) and (24), there are still two issues that must be resolved.

In the source term approach described in Section 4.5, one must decide how to split $\boldsymbol{\psi}(\boldsymbol{q}, \vec{x})$ into $\boldsymbol{\psi}^{1}(\boldsymbol{q}, \vec{x})$ and $\boldsymbol{\psi}^{2}(\boldsymbol{q}, \vec{x})$. We present in Sections 5.1 and 
5.2 a strategy based on geometric considerations for obtaining $\boldsymbol{\psi}^{1}(\boldsymbol{q}, \vec{x})$ and $\boldsymbol{\psi}^{2}(\boldsymbol{q}, \vec{x})$.

Another important issue is that we would much prefer to solve Riemann problems in an orthonormal frame instead of the coordinate basis in which equations (23) and (24) are written. In the coordinate basis, metric terms can complicate the eigenvectors and eigenvalues of the approximate flux Jacobian. The approximate flux Jacobian in an orthornormal or Cartesian frame is devoid of metric terms. We present in Section 5.3 a method for reducing the coordinate Riemann problems to locally valid orthonormal Riemann problems.

\subsection{Parallel transport}

In any type of Godunov scheme, one must solve a Riemann problem between two adjacent grid cells. A Riemann problem between two grid cells that lie on a curved manifold is complicated by the fact that the coordinate basis in which the solution is represented varies in space. For example, consider the two vectors $\boldsymbol{f}_{i j}^{1}$ and $\boldsymbol{f}_{i-1, j}^{1}$. Subtracting these two quantities, as is required in the wave decomposition (47), does not make sense since these vectors are being represented in different coordinate systems. To overcome this problem, one can transform vectors $\boldsymbol{f}_{i j}^{1}$ and $\boldsymbol{f}_{i-1, j}^{1}$ to a common coordinate system, for example the coordinate system at $\left(x_{i-1 / 2}^{1}, x_{j}^{2}\right)$, through the action of parallel transport [31].

Parallel transport is an operation that takes a vector $\vec{\mu}$ at a point $A$ and transports it to a point $B$ along the curve $\vec{c}(\lambda)$, where $\lambda$ is some parameterization of the curve (e.g., arclength). Parallel transport accomplishes this in such a way that $\vec{\mu}$ remains parallel to $\vec{c}(\lambda)$, or equivalently, the directional derivative of $\vec{c}(\lambda)$ in the direction of the tangent of $\vec{c}(\lambda)$ identically vanishes:

$$
\frac{d}{d \lambda} \vec{\mu} \equiv V^{k}\left(\nabla_{k} \mu^{j}\right) \vec{e}_{j} \equiv\left(\frac{d \mu^{j}}{d \lambda}+\Gamma_{m n}^{j} \mu^{m} V^{n}\right) \vec{e}_{j}=0
$$

where $\vec{V}=d \vec{c} / d \lambda$ is the tangent vector to $\vec{c}[31]$. For our purposes, we need to be able to transport vectors along coordinate lines, in which case (71) becomes

$$
\frac{\partial}{\partial x^{k}}\left[\begin{array}{c}
\mu^{1} \\
\mu^{2}
\end{array}\right]+\left[\begin{array}{cc}
\Gamma_{1 k}^{1} & \Gamma_{2 k}^{1} \\
\Gamma_{1 k}^{2} & \Gamma_{2 k}^{2}
\end{array}\right]\left[\begin{array}{l}
\mu^{1} \\
\mu^{2}
\end{array}\right]=0
$$

Equation (72) is a system of two coupled ordinary differential equations. Instead of computing the solution to this system exactly for each grid cell, we 
approximate the solution with a Taylor series:

$$
\begin{aligned}
\left.\vec{\mu}\right|_{x^{k}+\frac{\Delta x^{k}}{2}} & =\left.\vec{\mu}\right|_{x^{k}}+\left.\frac{\Delta x^{k}}{2} \frac{\partial}{\partial x^{k}} \vec{\mu}\right|_{x^{k}}+\mathcal{O}\left(\left(\Delta x^{k}\right)^{2}\right) \\
& =\left.\vec{\mu}\right|_{x^{k}}-\left.\frac{\Delta x^{k}}{2}\left[\begin{array}{ll}
\Gamma_{1 k}^{1} & \Gamma_{2 k}^{1} \\
\Gamma_{1 k}^{2} & \Gamma_{2 k}^{2}
\end{array}\right] \vec{\mu}\right|_{x^{k}}+\mathcal{O}\left(\left(\Delta x^{k}\right)^{2}\right)
\end{aligned}
$$

where there is no implied summation over $k$. Let $\boldsymbol{q}(\vec{x}, t)$ be the vector defined by (18). From the Taylor series, we can now define a parallel transport matrix that transforms the $\boldsymbol{q}(\vec{x}, t)$ from the coordinate basis at $x^{k}$ to the coordinate basis at $x^{k}+\Delta x^{k} / 2$ :

$$
\left[\begin{array}{c}
\rho \\
\mu^{1} \\
\mu^{2}
\end{array}\right]_{x^{k}+\frac{\Delta x^{n}}{2}} \approx\left(\mathcal{I} \mp \frac{\Delta x^{k}}{2}\left[\begin{array}{ccc}
0 & 0 & 0 \\
0 & \Gamma_{1 k}^{1} & \Gamma_{2 k}^{1} \\
0 & \Gamma_{1 k}^{2} & \Gamma_{2 k}^{2}
\end{array}\right]_{i j}\right)\left[\begin{array}{c}
\rho \\
\mu^{1} \\
\mu^{2}
\end{array}\right]_{x^{k}} \equiv P_{i j}^{k \pm}\left[\begin{array}{c}
\rho \\
\mu^{1} \\
\mu^{2}
\end{array}\right]_{x^{k}},
$$

where $\mathcal{I}$ is the identity matrix. This defines the parallel transport matrix $P_{i j}^{k \pm}$, where $i j$ is the grid point location, the $k$ superscript denotes the coordinate direction along which the propagation will occur $(k=1,2)$, and the \pm determines if the transport is in the positive $(+)$ or negative $(-)$ coordinate direction. To invert the parallel transport operation one can simply invert $P_{i j}^{k \pm}$ exactly.

\subsection{Parallel transport and the geometric source term}

From the geometric point view, parallel transport is a natural process in which two vectors that exist at different spatial locations can be expressed in the same reference frame. In this section we investigate what effect parallel transport has from the point of view of numerically solving the partial differential equations (23)-(24).

Let us consider the Riemann problem between the cells centered at $\left(x_{i}^{1}, x_{j}^{2}\right)$ and $\left(x_{i-1}^{1}, x_{j}^{2}\right)$. The fluxes in these cells are $\boldsymbol{f}_{i j}^{1}$ and $\boldsymbol{f}_{i-1, j}^{2}$, respectively. To compute the flux difference $\Delta \boldsymbol{F}^{1}$ required in the wave decomposition (47), we first apply the appropriate parallel transport matrices so that $\boldsymbol{f}_{i j}^{1}$ and $\boldsymbol{f}_{i-1, j}^{2}$ both get (approximately) represented in the coordinate basis at $\vec{x}_{i-1 / 2, j}$, then difference these two quantities:

$$
\Delta \boldsymbol{F}^{1}=P_{i j}^{1-} \boldsymbol{f}_{i j}^{1}-P_{i-1, j}^{1+} \boldsymbol{f}_{i-1, j}^{1}
$$


Using definition (74), this expression can be re-written as follows:

$$
\begin{aligned}
\Delta \boldsymbol{F}^{1} & =\left(\mathcal{I}+\frac{\Delta x^{1}}{2}\left[\begin{array}{ccc}
0 & 0 & 0 \\
0 & \Gamma_{11}^{1} & \Gamma_{21}^{1} \\
0 & \Gamma_{11}^{2} & \Gamma_{21}^{2}
\end{array}\right]_{i j}\right) \boldsymbol{f}_{i j}^{1}-\left(\mathcal{I}-\frac{\Delta x^{1}}{2}\left[\begin{array}{ccc}
0 & 0 & 0 \\
0 & \Gamma_{11}^{1} & \Gamma_{21}^{1} \\
0 & \Gamma_{11}^{2} & \Gamma_{21}^{2}
\end{array}\right]_{i-1, j}\right) \boldsymbol{f}_{i-1, j}^{1} \\
& =\boldsymbol{f}_{i j}^{1}-\boldsymbol{f}_{i-1, j}^{1}-\Delta x^{1} \boldsymbol{\psi}_{i-1 / 2, j}^{1},
\end{aligned}
$$

where

$$
\boldsymbol{\psi}_{i-1 / 2, j}^{1} \equiv-\frac{1}{2}\left(\left[\begin{array}{c}
0 \\
\sqrt{h}\left(\Gamma_{11}^{1} T^{11}+\Gamma_{12}^{1} T^{21}\right) \\
\sqrt{h}\left(\Gamma_{11}^{2} T^{11}+\Gamma_{12}^{2} T^{21}\right)
\end{array}\right]_{i j}+\left[\begin{array}{c}
0 \\
\sqrt{h}\left(\Gamma_{11}^{1} T^{11}+\Gamma_{12}^{1} T^{21}\right) \\
\sqrt{h}\left(\Gamma_{11}^{2} T^{11}+\Gamma_{12}^{2} T^{21}\right)
\end{array}\right]_{i-1, j}\right)
$$

This same analysis applied to a Riemann problem between the cells centered at $\left(x_{i}^{1}, x_{j}^{2}\right)$ and $\left(x_{i}^{1}, x_{j-1}^{2}\right)$ yields

$$
\Delta \boldsymbol{F}^{2}=\boldsymbol{f}_{i j}^{2}-\boldsymbol{f}_{i, j-1}^{2}-\Delta x^{2} \boldsymbol{\psi}_{i, j-1 / 2}^{2}
$$

where

$$
\boldsymbol{\psi}_{i, j-1 / 2}^{2} \equiv-\frac{1}{2}\left(\left[\begin{array}{c}
0 \\
\sqrt{h}\left(\Gamma_{21}^{1} T^{12}+\Gamma_{22}^{1} T^{22}\right) \\
\sqrt{h}\left(\Gamma_{21}^{2} T^{12}+\Gamma_{22}^{2} T^{22}\right)
\end{array}\right]_{i j}+\left[\begin{array}{c}
0 \\
\sqrt{h}\left(\Gamma_{21}^{1} T^{12}+\Gamma_{22}^{1} T^{22}\right) \\
\sqrt{h}\left(\Gamma_{21}^{2} T^{12}+\Gamma_{22}^{2} T^{22}\right)
\end{array}\right]_{i, j-1}\right)
$$

We showed in Section 4.5 that appropriately modifying the flux differences that are decomposed into waves yields a numerical method for solving balance laws. In particular, the modifications introduced into the flux differences by parallel transport yield a numerical update for a balance law with the following source term:

$$
\boldsymbol{\psi} \equiv \boldsymbol{\psi}^{1}+\boldsymbol{\psi}^{2}=-\sqrt{h}\left[\begin{array}{c}
0 \\
\Gamma_{n k}^{1} T^{k n} \\
\Gamma_{n k}^{2} T^{k n}
\end{array}\right] .
$$

This is precisely the source term found on the right hand side of equation (24) if we multiply (24) through by $\sqrt{h}$. We conclude from this analysis that the application of parallel transport to the flux functions produces a natural numerical method for dealing with the geometric source term in (24). To obtain a formally second order update we can again introduce correction terms of the form (66). 


\subsection{Orthonormalization}

For some hyperbolic partial differential equations, the Riemann problem in a general coordinate system may be significantly more difficult to solve than the Riemann problem for the same set of equations in an orthonormal frame. In this section we present a modification of the wave propagation method described so far that allows us to replace coordinate Riemann problems (23)(24) with locally valid orthonormal (Cartesian) Riemann problems. The approach we outline here was first introduced by Pons et al. [32] in the context of converting general relativistic Riemann problems into locally valid special relativistic Riemann problems.

If we again consider the model system (23)-(24), then the orthonormalization matrices that locally transform vectors in coordinate space to an orthonormal frame in the 1- and 2-directions are given by

$$
\begin{gathered}
\hat{\boldsymbol{Q}}=O^{1} \boldsymbol{Q}=\left[\begin{array}{ccc}
1 & 0 & 0 \\
0 & \frac{\sqrt{h_{22}}}{\sqrt{h}} & 0 \\
& & \\
0 & \frac{h_{12}}{\sqrt{h_{22}}} & \sqrt{h_{22}}
\end{array}\right] \boldsymbol{Q} \\
\hat{\boldsymbol{Q}}=O^{2} \boldsymbol{Q}=\left[\begin{array}{ccc}
1 & 0 & 0 \\
0 & \sqrt{h_{11}} & \frac{h_{12}}{\sqrt{h_{11}}} \\
0 & 0 & \frac{\sqrt{h_{11}}}{\sqrt{h}}
\end{array}\right] \boldsymbol{Q},
\end{gathered}
$$

respectively. A full derivation of these matrices can be found in [1].

In order to obtain an orthonormal Riemann problem, flux differences of the form (75) must be orthonormalized:

$$
\Delta \hat{\boldsymbol{F}}^{1}=O_{i-1 / 2, j}^{1}\left(P_{i j}^{1-} \boldsymbol{f}_{i j}^{1}-P_{i-1, j}^{1+} \boldsymbol{f}_{i-1, j}^{1}\right)
$$

For nonlinear problems we also need to compute the following quantities in order to construct an approximate flux Jacobian in the orthonormal frame:

$$
\begin{aligned}
& \hat{\boldsymbol{Q}}_{l}=O_{i-1 / 2, j}^{1} P_{i j}^{1-} \boldsymbol{Q}_{i j} \\
& \hat{\boldsymbol{Q}}_{r}=O_{i-1 / 2, j}^{1} P_{i-1, j}^{1+} \boldsymbol{Q}_{i-1, j}
\end{aligned}
$$

Once this has been accomplished, $\Delta \hat{\boldsymbol{F}}^{1}$ is decomposed into waves $\hat{\mathcal{Z}}^{p}$ and 
fluctuations are computed from these:

$$
\begin{aligned}
& \mathcal{A}_{1}^{+} \Delta \hat{\boldsymbol{Q}}=\sum_{p: \hat{s}^{p}>0} \hat{\mathcal{Z}}^{p}+\frac{1}{2} \sum_{p: \hat{s}^{p}=0} \hat{\mathcal{Z}}^{p} \\
& \mathcal{A}_{1}^{-} \Delta \hat{\boldsymbol{Q}}=\sum_{p: \hat{s}^{p}<0} \hat{\mathcal{Z}}^{p}+\frac{1}{2} \sum_{p: \hat{s}^{p}=0} \hat{\mathcal{Z}}^{p} .
\end{aligned}
$$

This information must now be de-orthonormalized in order for it to be used in the coordinate basis update:

$$
\begin{aligned}
s_{i-1 / 2, j}^{p} & =\hat{s}^{p} \cdot\left(\sqrt{h} \sqrt{h^{11}}\right)_{i-1 / 2, j} \\
\mathcal{Z}_{i-1 / 2, j}^{p} & =\left[O^{1}\right]_{i-1 / 2, j}^{-1} \hat{\mathcal{Z}}^{p} \\
\mathcal{A}_{1}^{ \pm} \Delta \boldsymbol{Q}_{i-1 / 2, j} & =\left[O^{1}\right]_{i-1 / 2, j}^{-1} \mathcal{A}_{1}^{ \pm} \Delta \hat{\boldsymbol{Q}} .
\end{aligned}
$$

These results are then used to construct high-resolution corrections (58). Finally, transverse Riemann problems are also solved in an orthonormal frame using the same technique. A more detailed description of the full wave propagation method on curved manifolds is presented in Section 6 .

\subsection{A note about accuracy and stability}

All the modifications presented in Section 4 maintain the order of accuracy and stability properties of the original wave propagation method. These modifications are each discussed in more detail elsewhere:

(1) Capacity form differencing (see [24,27]),

(2) Modified Riemann solvers for spatially varying flux functions (see [2]),

(3) Modified Riemann solvers for balance laws (see [2]), and

(4) Orthonormal Riemann solvers (see $[1,35]$ ).

\section{The algorithm}

In this section we outline in some detail our proposed wave propagation algorithm on curved manifolds. In each step it is understood that there is a loop over all indices $i$ and $j$ in the computational domain.

We propose the following algorithm: 
(1) Compute fluxes at cell centers:

$$
\boldsymbol{F}_{i j}^{1}=\boldsymbol{f}^{1}\left(\boldsymbol{Q}_{i j}^{n}, x_{i}^{1}, x_{j}^{2}\right)
$$

(2) Parallel transport the cell averages to the cell edges:

$$
\begin{aligned}
\boldsymbol{Q}_{r} & =P_{i j}^{1-} \boldsymbol{Q}_{i j}^{n} \\
\boldsymbol{Q}_{l} & =P_{i-1, j}^{1+} \boldsymbol{Q}_{i-1, j}^{n} \\
\Delta \overline{\boldsymbol{F}} & =P_{i j}^{1-} \boldsymbol{F}_{i j}^{1}-P_{i-1, j}^{1+} \boldsymbol{F}_{i-1, j}^{1} .
\end{aligned}
$$

(3) Orthonormalize at each cell edge:

$$
\begin{aligned}
\hat{\boldsymbol{Q}}_{r} & =\left[O^{1}\right]_{i-1 / 2, j} \boldsymbol{Q}_{r} \\
\hat{\boldsymbol{Q}}_{l} & =\left[O^{1}\right]_{i-1 / 2, j} \boldsymbol{Q}_{l} \\
\hat{\boldsymbol{Q}} & =\mathbf{A V G}\left(\hat{\boldsymbol{Q}}_{l}, \hat{\boldsymbol{Q}}_{r}\right) \\
\Delta \hat{\boldsymbol{F}} & =\left[O^{1}\right]_{i-1 / 2, j} \Delta \overline{\boldsymbol{F}}
\end{aligned}
$$

(4) Solve the normal Riemann problem by decomposing flux differences:

$$
\begin{aligned}
\hat{\mathcal{Z}}^{p} & =\left[\hat{\boldsymbol{\ell}}_{1}^{p} \cdot \Delta \hat{\boldsymbol{F}}\right] \hat{\boldsymbol{r}}_{1}^{p} \\
\mathcal{A}_{1}^{-} \Delta \hat{\boldsymbol{Q}} & =\sum_{p: \hat{s}^{p}<0} \hat{\mathcal{Z}}^{p}+\frac{1}{2} \sum_{p: \hat{s}^{p}=0} \hat{\mathcal{Z}}^{p} \\
\mathcal{A}_{1}^{+} \Delta \hat{\boldsymbol{Q}} & =\sum_{p: \hat{s}^{p}>0} \hat{\mathcal{Z}}^{p}+\frac{1}{2} \sum_{p: \hat{s}^{p}=0} \hat{\mathcal{Z}}^{p} .
\end{aligned}
$$

The above linearized Riemann problem is based on the eigenvalues $\left(\hat{s}^{p}\right)$, right eigenvectors $\left(\hat{\boldsymbol{r}}_{1}^{p}\right)$, and left eigenvectors $\left(\hat{\ell}_{1}^{p}\right)$ of the flux Jacobian evaluated at $\hat{\boldsymbol{Q}}$. $\hat{\boldsymbol{Q}}$ is obtained by consistently averaging $\hat{\boldsymbol{Q}}_{l}$ and $\hat{\boldsymbol{Q}}_{r}$; Roe averaging and arithmetic averaging are common choices.

(5) De-orthonormalize the results from the normal Riemann solve at the cell edge:

$$
\begin{aligned}
s_{i-1 / 2, j}^{p} & =\hat{s}^{p} \cdot\left(\sqrt{h} \sqrt{h^{11}}\right)_{i-1 / 2, j} \\
\mathcal{Z}_{i-1 / 2, j}^{p} & =\left[O^{1}\right]_{i-1 / 2, j}^{-1} \hat{\mathcal{Z}}^{p} \\
\mathcal{A}_{1}^{ \pm} \Delta \boldsymbol{Q}_{i-1 / 2, j} & =\left[O^{1}\right]_{i-1 / 2, j}^{-1} \mathcal{A}_{1}^{ \pm} \Delta \hat{\boldsymbol{Q}} .
\end{aligned}
$$


(6) Apply the TVD wave limiter:

$$
\begin{aligned}
\text { For each } p, \text { set } I & = \begin{cases}i-1 & \text { if } s_{i-1 / 2, j}^{p}>0 \\
i+1 & \text { if } s_{i-1 / 2, j}^{p}<0\end{cases} \\
\theta_{i-1 / 2, j}^{p} & =\frac{\mathcal{Z}_{I-1 / 2, j}^{p} \cdot \mathcal{Z}_{i-1 / 2, j}^{p}}{\mathcal{Z}_{i-1 / 2, j}^{p} \cdot \mathcal{Z}_{i-1 / 2, j}^{p}} \\
\tilde{\mathcal{Z}}_{i-1 / 2, j}^{p} & =\mathcal{Z}_{i-1 / 2, j}^{p} \phi\left(\theta_{i-1 / 2, j}^{p}\right) .
\end{aligned}
$$

(7) Construct high-resolution corrections:

$$
\tilde{\boldsymbol{F}}_{i-1 / 2, j}^{1}=\frac{1}{2} \sum_{p=1}^{M} \operatorname{sign}\left(s_{i-1 / 2, j}^{p}\right)\left(1-\frac{1}{\sqrt{h_{i-1 / 2, j}}} \frac{\Delta t}{\Delta x^{1}}\left|s_{i-1 / 2, j}^{p}\right|\right) \tilde{\mathcal{Z}}_{i-1 / 2, j}^{p} .
$$

(8) Construct second order source term corrections:

$$
\boldsymbol{S}_{i j}^{1}=\frac{1}{2}\left[\frac{\partial\left(\boldsymbol{\psi}^{1}+\boldsymbol{\psi}^{2}\right)}{\partial \boldsymbol{q}}\right]_{i j} \sum_{p=1}^{M}\left[\tilde{\mathcal{Z}}_{i-1 / 2, j}^{p}+\tilde{\mathcal{Z}}_{i+1 / 2, j}^{p}\right]
$$

(9) Add high-resolution corrections to fluctuations and orthonormalize the result at the cell edge $\left(\boldsymbol{Q}_{l}\right.$ and $\boldsymbol{Q}_{r}$ are from Step (2)):

$$
\begin{aligned}
\hat{\boldsymbol{Q}}_{r} & =\left[O^{2}\right]_{i-1 / 2, j} \boldsymbol{Q}_{r} \\
\hat{\boldsymbol{Q}}_{l} & =\left[O^{2}\right]_{i-1 / 2, j} \boldsymbol{Q}_{l} \\
\hat{\boldsymbol{Q}} & =\operatorname{AVG}\left(\hat{\boldsymbol{Q}}_{l}, \hat{\boldsymbol{Q}}_{r}\right) \\
\mathcal{A}_{1}^{ \pm \Delta} \hat{\boldsymbol{Q}} & =\left[O^{2}\right]_{i-1 / 2, j}\left(\boldsymbol{\mathcal { A }}_{1}^{ \pm} \Delta \boldsymbol{Q}_{i-1 / 2, j} \mp 2 \boldsymbol{F}_{i-1 / 2, j}^{1}\right) .
\end{aligned}
$$

(10) Solve transverse Riemann problem at the cell edge:

$$
\begin{aligned}
\beta_{-}^{p} & =\hat{\boldsymbol{\ell}}_{2}^{p} \cdot \mathcal{A}_{1}^{-} \Delta \hat{\boldsymbol{Q}} \\
\beta_{+}^{p} & =\hat{\boldsymbol{\ell}}_{2}^{p} \cdot \mathcal{A}_{1}^{+} \Delta \hat{\boldsymbol{Q}} \\
\mathcal{A}_{2}^{-} \mathcal{A}_{1}^{-} \Delta \hat{\boldsymbol{Q}} & =\sum_{p: \hat{s}^{p}<0} \hat{s}^{p} \beta_{-}^{p} \hat{\boldsymbol{r}}_{2}^{p} \\
\mathcal{A}_{2}^{-} \mathcal{A}_{1}^{+} \Delta \hat{\boldsymbol{Q}} & =\sum_{p: \hat{s}^{p}<0} \hat{s}^{p} \beta_{+}^{p} \hat{\boldsymbol{r}}_{2}^{p} \\
\mathcal{A}_{2}^{+} \mathcal{A}_{1}^{-} \Delta \hat{\boldsymbol{Q}} & =\sum_{p: \hat{s}^{p}>0} \hat{s}^{p} \beta_{-}^{p} \hat{\boldsymbol{r}}_{2}^{p} \\
\mathcal{A}_{2}^{+} \mathcal{A}_{1}^{+} \Delta \hat{\boldsymbol{Q}} & =\sum_{p: \hat{s}^{p}>0} \hat{s}^{p} \beta_{+}^{p} \hat{\boldsymbol{r}}_{2}^{p} .
\end{aligned}
$$


In the above expressions, $\hat{s}^{p}, \hat{\boldsymbol{r}}_{2}^{p}$, and $\hat{\boldsymbol{\ell}}_{2}^{p}$ are the eigenvalues, right eigenvectors, and left eigenvectors of the approximate flux Jacobian in the 2-direction. These quantities are evaluated at $\hat{\boldsymbol{Q}}$, where $\hat{\boldsymbol{Q}}$ is the same quantity as computed in step (3).

(11) De-orthonormalize at the cell edge:

$$
\begin{aligned}
& \mathcal{A}_{2}^{ \pm} \mathcal{A}_{1}^{-} \Delta \boldsymbol{Q}_{i-1 / 2, j}=\left(\sqrt{h} \sqrt{h^{22}}\right)_{i-1 / 2, j}\left[O^{2}\right]_{i-1 / 2, j}^{-1} \mathcal{A}_{2}^{ \pm} \mathcal{A}_{1}^{-} \Delta \hat{\boldsymbol{Q}} \\
& \mathcal{A}_{2}^{ \pm} \mathcal{A}_{1}^{+} \Delta \boldsymbol{Q}_{i-1 / 2, j}=\left(\sqrt{h} \sqrt{h^{22}}\right)_{i-1 / 2, j}\left[O^{2}\right]_{i-1 / 2, j}^{-1} \mathcal{A}_{2}^{ \pm} \mathcal{A}_{1}^{+} \Delta \hat{\boldsymbol{Q}}
\end{aligned}
$$

(12) Correct fluxes:

$$
\begin{gathered}
\tilde{\boldsymbol{F}}_{i, j+1 / 2}^{2}=\tilde{\boldsymbol{F}}_{i, j+1 / 2}^{2}-\frac{1}{2} \frac{1}{\sqrt{h_{i-1 / 2, j}}} \frac{\Delta t}{\Delta x^{1}} \mathcal{A}_{2}^{+} \mathcal{A}_{1}^{+} \Delta \boldsymbol{Q}_{i-1 / 2, j} \\
\tilde{\boldsymbol{F}}_{i, j-1 / 2}^{2}=\tilde{\boldsymbol{F}}_{i, j-1 / 2}^{2}-\frac{1}{2} \frac{1}{\sqrt{h_{i-1 / 2, j}}} \frac{\Delta t}{\Delta x^{1}} \mathcal{A}_{2}^{-} \mathcal{A}_{1}^{+} \Delta \boldsymbol{Q}_{i-1 / 2, j} \\
\tilde{\boldsymbol{F}}_{i-1, j+1 / 2}^{2}=\tilde{\boldsymbol{F}}_{i-1, j+1 / 2}^{2}-\frac{1}{2} \frac{1}{\sqrt{h_{i-1 / 2, j}}} \frac{\Delta t}{\Delta x^{1}} \mathcal{A}_{2}^{+} \mathcal{A}_{1}^{-} \Delta \boldsymbol{Q}_{i-1 / 2, j} \\
\tilde{\boldsymbol{F}}_{i-1, j-1 / 2}^{2}=\tilde{\boldsymbol{F}}_{i-1, j-1 / 2}^{2}-\frac{1}{2} \frac{1}{\sqrt{h_{i-1 / 2, j}}} \frac{\Delta t}{\Delta x^{1}} \mathcal{A}_{2}^{-} \mathcal{A}_{1}^{-} \Delta \boldsymbol{Q}_{i-1 / 2, j}
\end{gathered}
$$

(13) Repeat steps (1)-(12) in the 2-direction.

(14) Update solution:

$$
\begin{aligned}
\boldsymbol{Q}_{i j}^{n+1}=\boldsymbol{Q}_{i j}^{n} & -\frac{1}{2} \frac{1}{\sqrt{h_{i j}}} \frac{\Delta t^{2}}{\Delta x^{1}} \boldsymbol{S}_{i j}^{1}-\frac{1}{2} \frac{1}{\sqrt{h_{i j}}} \frac{\Delta t^{2}}{\Delta x^{2}} \boldsymbol{S}_{i j}^{2} \\
- & \frac{1}{\sqrt{h_{i j}}} \frac{\Delta t}{\Delta x^{1}}\left[\mathcal{A}_{1}^{-} \Delta \boldsymbol{Q}_{i+1 / 2, j}+\mathcal{A}_{1}^{+} \Delta \boldsymbol{Q}_{i-1 / 2, j}\right] \\
- & \frac{1}{\sqrt{h_{i j}}} \frac{\Delta t}{\Delta x^{2}}\left[\mathcal{A}_{2}^{-} \Delta \boldsymbol{Q}_{i, j+1 / 2}+\mathcal{A}_{2}^{+} \Delta \boldsymbol{Q}_{i, j-1 / 2}\right] \\
- & \frac{1}{\sqrt{h_{i j}}} \frac{\Delta t}{\Delta x^{1}}\left[\tilde{\boldsymbol{F}}_{i+1 / 2, j}^{1}-\tilde{\boldsymbol{F}}_{i-1 / 2, j}^{1}\right] \\
- & \frac{1}{\sqrt{h_{i j}}} \frac{\Delta t}{\Delta x^{2}}\left[\tilde{\boldsymbol{F}}_{i, j+1 / 2}^{2}-\tilde{\boldsymbol{F}}_{i, j-1 / 2}^{2}\right] .
\end{aligned}
$$

The above stated algorithm has been implemented in Fortran and is freely available as part of the CLAWPACK software package, as an extension called CLAWMAN; see

http: //www . amath. washington. edu/ claw/clawman.html. 
The latest version of CLAWPACK also includes MATLAB graphics routines for visualizing solutions on two-dimensional manifolds.

In the standard CLAWPACK software package, the user must specify initial conditions (qinit.f), boundary conditions (bc2.f), and a normal and a transverse Riemann solver (rpn2.f and rpt2.f). The only additional information that the user must specify in the curved manifold extension is a subroutine that specifies the metric tensor (metric.f), the flux function (flux fun2.f), and the matrix $\partial \boldsymbol{\psi} / \partial \boldsymbol{q}$ (psiq2.f). All the required operations of parallel transport, orthonormalization, and re-scaling are handled internally by the algorithm.

\section{The scalar field equation}

In the remainder of the paper we present some numerical test problems to demonstrate the accuracy of this algorithm. More results, some animations, and the source code may be found at

http://www . amath. washington. edu/ claw/clawman.html.

As a first example, we apply the wave propagation algorithm to the scalar field equation on a curved manifold $\mathcal{M}$. This equation models the propagation of acoustic waves in a thin membrane whose shape is given by the manifold $\mathcal{M}$. The scalar field equation can be written as

$$
\frac{\partial^{2}}{\partial t^{2}} \varphi-\vec{\nabla} \cdot(\vec{\nabla} \varphi)=0
$$

The pressure, $p(\vec{x}, t)$, and the fluid velocity, $\vec{u}(\vec{x}, t)$, can be obtained by taking appropriate temporal and spatial gradients of the scalar field:

$$
\begin{aligned}
& p(\vec{x}, t)=-\frac{\partial}{\partial t} \varphi(\vec{x}, t) \\
& \vec{u}(\vec{x}, t)=\vec{\nabla} \varphi(\vec{x}, t) .
\end{aligned}
$$

Replacing $\varphi(\vec{x}, t)$ in equation (91) by the above definitions and imposing that

$$
\vec{\nabla}\left(\frac{\partial}{\partial t} \varphi\right)=\frac{\partial}{\partial t}(\vec{\nabla} \varphi)
$$

results in the following system of balance laws for the pressure and the components of the fluid velocity:

$$
\frac{\partial}{\partial t} \boldsymbol{q}+\frac{1}{\sqrt{h}} \frac{\partial}{\partial x^{k}} \boldsymbol{f}^{k}=\boldsymbol{\psi}_{c}
$$


where

$$
\boldsymbol{q}(\vec{x}, t)=\left[\begin{array}{c}
p \\
u^{1} \\
u^{2}
\end{array}\right], \quad \boldsymbol{f}^{k}(\boldsymbol{q}, \vec{x})=\sqrt{h}\left[\begin{array}{c}
u^{k} \\
p h^{k 1} \\
p h^{k 2}
\end{array}\right], \quad \boldsymbol{\psi}_{c}(\boldsymbol{q}, \vec{x})=\left[\begin{array}{c}
0 \\
-\Gamma_{m n}^{1} p h^{n m} \\
-\Gamma_{m n}^{2} p h^{n m}
\end{array}\right] .
$$

The orthonormal version of equation (96) can be obtained by setting $\overleftrightarrow{h}$ to the identity matrix. The corresponding wave decomposition of the orthonormal flux Jacobian is

$$
\begin{aligned}
& \hat{\boldsymbol{R}}_{1}=\left[\begin{array}{rrr}
-1 & 0 & 1 \\
1 & 0 & 1 \\
0 & 1 & 0
\end{array}\right], \hat{\boldsymbol{\Lambda}}_{1}=\left[\begin{array}{ll}
-1 & \\
& 0 \\
& 1
\end{array}\right], \hat{\boldsymbol{R}}_{1}^{-1}=\frac{1}{2}\left[\begin{array}{ccc}
-1 & 1 & 0 \\
0 & 0 & 2 \\
1 & 1 & 0
\end{array}\right], \\
& \hat{\boldsymbol{R}}_{2}=\left[\begin{array}{lll}
-1 & 0 & 1 \\
0 & 1 & 0 \\
1 & 0 & 1
\end{array}\right], \hat{\boldsymbol{\Lambda}}_{2}=\left[\begin{array}{cc}
-1 & \\
& 0 \\
& 1
\end{array}\right], \hat{\boldsymbol{R}}_{2}^{-1}=\frac{1}{2}\left[\begin{array}{ccc}
-1 & 0 & 1 \\
0 & 2 & 0 \\
1 & 0 & 1
\end{array}\right] .
\end{aligned}
$$

One can easily show that the orthonormal equations form a strictly hyperbolic system of conservation laws (see LeVeque [27]).

\subsection{The geometry}

We consider the propagation of sound waves on a surface given by $z=b(x, y)$, where $x, y$, and $z$ are the standard Cartesian coordinates. In this case, the transformation to surface coordinates is quite simple since $x$ and $y$ already parameterize the surface:

$$
\begin{aligned}
& x=X\left(x^{1}, x^{2}\right)=x^{1} \\
& y=Y\left(x^{1}, x^{2}\right)=x^{2} \\
& z=Z\left(x^{1}, x^{2}\right)=b\left(x^{1}, x^{2}\right) .
\end{aligned}
$$

The Jacobian matrix that transforms a vector on the surface of $\mathcal{M}$ into a Cartesian vector is

$$
J=\left[\begin{array}{cc}
1 & 0 \\
0 & 1 \\
\frac{\partial b}{\partial x^{1}} & \frac{\partial b}{\partial x^{2}}
\end{array}\right]
$$


The metric and the square root of the determinant of the metric for this coordinate system are

$$
\begin{gathered}
\overleftrightarrow{h}=\left[\begin{array}{cc}
1+\left(\frac{\partial b}{\partial x^{1}}\right)^{2} & \frac{\partial b}{\partial x^{1}} \frac{\partial b}{\partial x^{2}} \\
\frac{\partial b}{\partial x^{1}} \frac{\partial b}{\partial x^{2}} & 1+\left(\frac{\partial b}{\partial x^{2}}\right)^{2}
\end{array}\right] \\
\sqrt{h}=\sqrt{1+\left(\frac{\partial b}{\partial x^{1}}\right)^{2}+\left(\frac{\partial b}{\partial x^{2}}\right)^{2}}
\end{gathered}
$$

respectively. In the example shown below we set

$$
b(r)=-\exp \left[-\left(\frac{r}{0.22}\right)^{2}\right]
$$

where

$$
r=\sqrt{\left(x^{1}\right)^{2}+\left(x^{2}\right)^{2}}
$$

is the radial distance from the origin to the point $\left(x^{1}, x^{2}\right)$.

Note that this manifold is asymptotically flat and that the coordinate basis is asymptotically orthogonal as $r \rightarrow \infty$. Near the orgin, however, the manifold is strongly curved and the coordinate basis is non-orthogonal. This surface is shown in Figure 3.

\subsection{Test 1: order of accuracy}

We begin by considering an examples in which the initial conditions are radially symmetric:

$$
\begin{gathered}
p(r, 0)= \begin{cases}0.5 \cos \left(\frac{20 \pi}{3}(r-0.35)\right)+0.5 & \text { if } \quad 0.2 \leq r \leq 0.5 \\
0 & \text { otherwise }\end{cases} \\
u^{1}(r, 0)=u^{2}(r, 0)=0 .
\end{gathered}
$$

The magnitude of the velocity vector in $\mathbb{R}^{3}$ is given by

$$
\left|\vec{u}_{3 D}\right|=\sqrt{\left(u^{1}\right)^{2}+\left(u^{2}\right)^{2}+\left(u^{1} \frac{\partial b}{\partial x^{1}}+u^{2} \frac{\partial b}{\partial x^{2}}\right)^{2}}
$$

Because the initial conditions and the metric tensor are radially symmetric, the exact solution in this example is also radially symmetric. In order to compute the order of accuracy of the wave propagation method, we compare the curved manifold solution to a highly resolved (6000 points) one-dimensional 
the radially symmetric equations,

$$
\begin{aligned}
\frac{\partial}{\partial t} p+\frac{\partial}{\partial r}\left(h^{r r} v\right) & =-\frac{h^{r r} v}{\sqrt{h}} \frac{\partial}{\partial r}(\sqrt{h}) \\
\frac{\partial}{\partial t} v+\frac{\partial}{\partial r} p & =0
\end{aligned}
$$

where

$$
\begin{aligned}
\sqrt{h} & =r \sqrt{1+\left(b^{\prime}(r)\right)^{2}} \\
h^{r r} & =\left(1+\left(b^{\prime}(r)\right)^{2}\right)^{-1} \\
\left|\vec{u}_{3 D}\right| & =\sqrt{h^{r r} v^{2}} .
\end{aligned}
$$

The computed pressure and magnitude of velocity at time $t=0.45$ are shown in Figure 1(a) and 1(b), respectively. These graphs are scatter plots of the curved manifold solution on a $100 \times 100$ grid along with a highly resolved onedimensional solution. The results demonstrate the ability of the wave propagation algorithm to accurately approximate the solution and maintain the radial symmetry of the problem. Direct experimental verification of second order accuracy is shown in Figure 2. These plots clearly indicate that the wave propagation method is $\mathcal{O}\left(|\Delta \vec{x}|^{2}, \Delta t^{2}\right)$ in both pressure and velocity.

\subsection{Test 2: multidimensional wave}

Next we consider a non-symmetric solution to the acoustic equations. The initial conditions for this test consist of a right-going pressure pulse started near the left edge of the computational domain:

$$
\begin{aligned}
p(x, y, 0) & = \begin{cases}1 & \text { if }-0.95<x<-0.75 \\
0 & \text { otherwise }\end{cases} \\
u^{1}(r, 0) & = \begin{cases}1 & \text { if }-0.95<x<-0.75 \\
0 & \text { otherwise }\end{cases} \\
u^{2}(r, 0) & =0
\end{aligned}
$$

The solution with these initial conditions on a $200 \times 200$ grid is shown in Figure 3. The same problem on a $400 \times 400$ grid is shown in Figure 4 . On the solution we superimpose the curved manifold, $z=b(x, y)$, but sampled at a lower resolution of $50 \times 50$. This result demonstrates the ability of the wave propagation algorithm to resolve discontinuous solutions in multidimensions. 


\section{Shallow water flow on the sphere}

Geophysical fluid dynamics is another application where it is important to solve hyperbolic systems on curved manifolds. The shallow water equations on the surface of a sphere provide a simplified model for the dynamics of the Earth's global atmosphere. A more sophisticated model would be to consider the muliti-layer shallow water equations. In either case, the Earth's curvature affects the dynamics of the atmosphere.

An important first step in developing a dynamical core for global climate model (GCM) is to consider the solution of the shallow water equations on the surface of a sphere [44].

The shallow water equations in generalized coordinates satisfy equation (95) with:

$$
\boldsymbol{q}(\vec{x}, t)=\left[\begin{array}{c}
\phi \\
\phi u^{1} \\
\phi u^{2}
\end{array}\right], \quad \boldsymbol{f}^{k}(\boldsymbol{q}, \vec{x})=\sqrt{h}\left[\begin{array}{c}
\phi u_{k} \\
T^{k 1} \\
T^{k 2}
\end{array}\right], \quad \boldsymbol{\psi}_{c}(\boldsymbol{q}, \vec{x})=\left[\begin{array}{c}
0 \\
-\Gamma_{m n}^{1} T^{n m} \\
-\Gamma_{m n}^{2} T^{n m}
\end{array}\right],
$$

and

$$
T^{n m}=\phi u^{n} u^{m}+\frac{1}{2} \phi^{2} h^{n m} .
$$

A full derivation of the shallow water equations in Cartesian coordinates can be found in Kevorkian [20].

The wave decomposition of the flux Jacobians is given below:

$$
\begin{aligned}
& \hat{\boldsymbol{R}}_{1}=\left[\begin{array}{ccc}
1 & 0 & 1 \\
\hat{U}^{1}-\sqrt{\Phi} & 0 & \hat{U}^{1}+\sqrt{\Phi} \\
\hat{U}^{2} & 1 & \hat{U}^{2}
\end{array}\right], \hat{\boldsymbol{\Lambda}}_{1}=\left[\begin{array}{ccc}
\hat{U}^{1}-\sqrt{\Phi} & 0 & 0 \\
0 & \hat{U}^{1} & 0 \\
0 & 0 & \hat{U}^{1}+\sqrt{\Phi}
\end{array}\right] \\
& \hat{\boldsymbol{R}}_{2}=\left[\begin{array}{ccc}
1 & 0 & 1 \\
\hat{U}^{1} & 1 & \hat{U}^{1} \\
\hat{U}^{2}-\sqrt{\Phi} & 0 & \hat{U}^{2}+\sqrt{\Phi}
\end{array}\right], \hat{\Lambda}_{2}=\left[\begin{array}{ccc}
\hat{U}^{2}-\sqrt{\Phi} & 0 & 0 \\
0 & \hat{U}^{2} & 0 \\
0 & 0 & \hat{U}^{2}+\sqrt{\Phi}
\end{array}\right] \text {, }
\end{aligned}
$$

where

$$
\begin{aligned}
\Phi & =\frac{1}{2}\left(\phi_{r}+\phi_{l}\right) \\
\hat{U}^{k} & =\frac{\sqrt{\phi_{r}} \hat{u}_{r}^{k}+\sqrt{\phi_{l}} \hat{u}_{l}^{k}}{\sqrt{\phi_{r}}+\sqrt{\phi_{l}}}
\end{aligned}
$$


are the Roe averages and $\left(\phi_{l}, \hat{u}_{l}^{1}, \hat{u}_{l}^{2}\right)$ and $\left(\phi_{r}, \hat{u}_{r}^{1}, \hat{u}_{r}^{2}\right)$ are the constant initial states on the left and right, respectively. If a transonic rarefaction is encountered in the Riemann problem we construct fluctuations based on the Local Lax-Friedrichs (LLF) method [36]. The reader is refered to [27] for a discussion of Roe averages and entropy fixes.

\subsection{The geometry}

Although there are many types of numerical grids that have been devised to solve the shallow water equations on the surface of a sphere $[14,15,30,33,34,37]$, we consider in this paper only a longitude-latitude grid. In order to avoid the coordinate singularities that occur at the North and South Poles, we will only compute solutions on part of the sphere.

The numerical method presented in this paper has been successfully applied to the "cubed sphere" in [35], which appears to be a much better choice of coordinates for problems on the full sphere. In this paper we do not make use of the "cubed sphere" grid primarily because we would like to avoid discussion of the complications resulting from internal boundaries and "corner" points; and instead, our focus here will be on the affects of curvature on the shallow water equations. See [35] for a treatment of the internal boundaries and "corner" points arising from the "cubed sphere" grid and some shallow water results on the full sphere.

Let $\left(x^{1}, x^{2}\right) \in\left[-180^{\circ}, 180^{\circ}\right] \times\left[-90^{\circ}, 90^{\circ}\right]$ be the longitude and latitude, respectively, on a sphere of radius $r$. The coordinate transformation from the sphere to Cartesian coordinates is

$$
\begin{aligned}
& x=X\left(x^{1}, x^{2}\right)=r \cos \left(x^{2}\right) \cos \left(x^{1}\right) \\
& y=Y\left(x^{1}, x^{2}\right)=r \cos \left(x^{2}\right) \sin \left(x^{1}\right) \\
& z=Z\left(x^{1}, x^{2}\right)=r \sin \left(x^{2}\right) .
\end{aligned}
$$

The metric and the square root of the determinant of the metric for this coordinate system are

$$
\begin{aligned}
\overleftrightarrow{h} & =\left[\begin{array}{cc}
r^{2} \cos ^{2}\left(x^{2}\right) & 0 \\
0 & r^{2}
\end{array}\right] \\
\sqrt{h} & =r^{2} \cos \left(x^{2}\right),
\end{aligned}
$$

respectively. In all the computations below we set $r=1$. 


\subsection{Test 1: non-rotating sphere}

We first consider shallow water flow on a non-rotating sphere. In order to avoid the coordinate singularities at the poles, the shallow water equations are solved on only part of sphere: $\left(x^{1}, x^{2}\right) \in\left[-90^{\circ}, 90^{\circ}\right] \times\left[-75^{\circ}, 75^{\circ}\right]$. For the initial conditions we place a "circular" depth disturbance at the equator:

$$
\begin{aligned}
\phi\left(x^{1}, x^{2}, 0\right) & = \begin{cases}2 & \text { if } \cos ^{-1}\left(\cos \left(x^{1}\right) \cos \left(x^{2}\right)\right) \leq 0.2 \\
0.2 & \text { otherwise }\end{cases} \\
u^{1}\left(x^{1}, x^{2}, 0\right) & =u^{2}\left(x^{1}, x^{2}, 0\right)=0 .
\end{aligned}
$$

This initial condition is symmetric about the point $\left(x^{1}, x^{2}\right)=\left(0^{\circ}, 0^{\circ}\right)$; and therefore, the solution should remain symmetric for all time. After imposing symmetry, the shallow water equations reduce to the system

$$
\frac{\partial}{\partial t}\left[\begin{array}{c}
\phi \\
\phi v
\end{array}\right]+\frac{\partial}{\partial \theta}\left[\begin{array}{c}
\phi v \\
\phi v^{2}+\frac{1}{2} \phi^{2}
\end{array}\right]=\tan (\theta)\left[\begin{array}{c}
\phi v \\
\phi v^{2}
\end{array}\right]
$$

where $\theta$ is an angle measured from $\left(0^{\circ}, 0^{\circ}\right)$ and $v$ is the angular velocity away from $\left(0^{\circ}, 0^{\circ}\right)$.

The solution to the multidimensional equations on a $204 \times 170$ grid at various times is shown in Figure 5. A scatter plot of this data along with a highly resolved (2000 points) solution of (125) is shown in Figure 6.

\subsection{Test 2: rotating sphere}

Finally, we consider the shallow water equations on a rotating sphere. The rotation of the Earth is an important element in the dynamics of the atmosphere and ocean. Without loss of generality we let the axis of rotation be the Cartesian $z$-axis. If we solve the shallow water equations in the reference frame of the rotating Earth, we introduce in the momentum equations a pseudo-force of the form

$$
-2 \Omega \vec{k} \times \vec{u}-\Omega \vec{k} \times(\Omega \vec{k} \times \vec{r}),
$$

where $\Omega$ is the rotation rate, $\vec{k}$ is the unit vector in the $z$-direction, and $\vec{r}$ is the position vector (i.e., $\vec{r}=(x, y, z)$ in Cartesian coordinates). The first term in the above expression is called the Coriolis force and the second term is the centrifugal force. In order to keep matter from flying off of the surface of the Earth, the centrifugal force and the gravitational force must be in balance. Therefore, for flows that are confined to the surface of the Earth only the 
Coriolis force will affect the dynamics and we can write:

$$
\psi_{f} \equiv-f \vec{k} \times \vec{u},
$$

where $f=2 \Omega$ is the Coriolis parameter [6]. The Coriolis force written in spherical coordinates is given by:

$$
\boldsymbol{\psi}_{f}=f \tan \left(x^{2}\right)\left[\begin{array}{c}
\phi u^{2} \\
-\cos ^{2}\left(x^{2}\right) \phi u^{1}
\end{array}\right] .
$$

The rotating shallow water equations differ from the non-rotating equations only in that the source term, $\boldsymbol{\psi}_{c}$, is modified as follows:

$$
\boldsymbol{\psi}_{c}(\boldsymbol{q}, \vec{x})=\left[\begin{array}{c}
0 \\
-\Gamma_{m n}^{1} T^{n m} \\
-\Gamma_{m n}^{2} T^{n m}
\end{array}\right]+f \tan \left(x^{2}\right)\left[\begin{array}{c}
0 \\
\phi u^{2} \\
-\cos ^{2}\left(x^{2}\right) \phi u^{1}
\end{array}\right] .
$$

In terms of the wave propagation algorithm, we handle the geometric source term through parallel transport and the Coriolis force through Strang operator splitting (see Chapter 17 of [27]). The results of computation on a $204 \times 170$ grid using initial conditions (123) and (124) and a Coriolis parameter of $f=10$ are shown in Figure 7. A fine grid calculation on a $408 \times 340$ grid is shown in Figure 8.

\section{Conclusions}

We have presented in this a paper a generalization of the wave propagation method for hyperbolic systems on curved manifolds [23,24,27]. A slightly different version of the method presented in this paper was first considered in $[1,35]$. The equations are solved in a coordinate basis resulting from the choice of coordinates on the manifold. In general the coordinate basis representation produces a set of balance laws in which flux functions explicitly vary in space and source terms due to geometric effects are present. The explicit spatial variation is handled through an approach introduced in [2] in which linearized Riemann problems are solved by locally decomposing flux differences. The geometric source terms are handled through the action of parallel transport. The process of parallel transport is shown to be mathematically equivalent to a procedure described in $[2,28]$ in which source terms are incorporated into Riemann problems. Therefore, the resulting wave propagation method does not require the use of operator splitting techniques. 
We argue that the wave propagation method is second order accurate for smooth solutions as well as shock-capturing. These claims are experimentally verified by using the algorithm to compute the solution to the acoustic equations on a curved manifold as well as the solution of the shallow water equations on a sphere. The Fortran code that was used to obtain these solutions is freely available for download as an add-on to the standard CLAWPACK software package.

The proposed wave propagation method has potential applications in several areas including large-scale atmospheric flows, solar magnetohydrodynamics, and relativistic hydrodynamics. Some preliminary work on applying this method to relativistic hydrodynamics can be found in [1] and to shallow water and shallow water magnetohydrodynamic flow on the sphere in [35]. In addition to these applications, future work will also concentrate on developing an adaptive mesh refinement (AMR) version of the CLAWMAN software.

Acknowledgements. This work was supported in part by NSF grants DMS9803442, DMS-9810726, DMS-0106511, and DMS-9977371 and DOE grants DE-FG03-96ER25292 and DE-FC02-01ER25474.

\section{References}

[1] D.S. Bale. Wave propagation algorithms on curved manifolds with applications to relativistic hydrodynamics. PhD thesis, University of Washington, Seattle, Washington, 2002.

[2] D.S. Bale, R.J. LeVeque, S. Mitran, and J.A. Rossmanith. A wave propagation method for conservation laws and balance laws with spatially varying flux functions. SIAM J. Sci. Comp., 24:955-978, 2003.

[3] J.M. Bardeen and L.T. Buchman. Numerical tests of evolution systems, gauge conditions, and boundary conditions for 1d colliding gravitational plane waves. Phys. Rev. D, 65, 2002.

[4] J.Y-K. Cho and L.M. Polvani. The emergence of jets and vortices in freely evolving, shallow-water turbulence on a sphere. Physics of Fluids, 8:1531-1552, 1995.

[5] J. Coté. A Lagrange multiplier approach for the metric terms of semiLangrangian models on the sphere. Q.J.R. Meteorol. Soc., 114:1347-1352, 1988.

[6] B. Cushman-Roisin. Introduction to Geophysical Fluid Dynamics. Prentice Hall, 1994.

[7] R. Donat and A. Marquina. Capturing shock reflections: an improved flux formula. J. Comp. Phys., 125:42-58, 1996. 
[8] T. Fogarty. Finite volume methods for acoustics and elasto-plasticity with damage in a heterogeneous medium. PhD thesis, University of Washington, Seattle, Washington, 2002.

[9] T.R. Fogarty. High-resolution finite volume methods for acoustics in a rapidlyvarying heterogeneous medium. Master's thesis, University of Washington, Seattle, Washington, 1997.

[10] T.R. Fogarty and R.J. LeVeque. High-resolution finite volume methods for acoustics in periodic and random media. Journal of the Acoustical Society of America, 106(1):1-12, July 1999.

[11] J.A. Font. Numerical hydrodynamics in general relativity. Living Rev. Rel., 2000 .

[12] P.A. Gilman. Magnetohydrodynamic "shallow water" equations for the solar tachocline. Astrophys. J., 544:L79-L82, 2000.

[13] A. Harten and J.M. Hyman. Self-adjusting grid methods for one-dimensional hyperbolic conservation laws. J. Comp. Phys., 50:235-269, 1983.

[14] R. Heikes and D.A. Randall. Numerical integration integration of the shallow water equations on a twisted icosahedral grid. Part I: Basic design and results of tests. Monthly Weather Review, 123:1862-1880, 1995.

[15] R. Heikes and D.A. Randall. Numerical integration integration of the shallow water equations on a twisted icosahedral grid. Part II: A detailed description of the grid and an analysis of numerical accuracy. Monthly Weather Review, 123:1881-1887, 1995.

[16] C. Helzel. Numerical approximation of conservation laws with stiff source terms for the modelling of detonation waves. $\mathrm{PhD}$ thesis, Otto-von-GuerickeUniversität Magdeburg, Magdeburg,Germany, 2000.

[17] C. Helzel, R.J. LeVeque, and G. Warnecke. A modified fractional step method for the accurate approximation of detonation waves. SIAM J. Sci. Comp., $22: 1489-1510,2000$.

[18] S. D. Hern and J. M. Stewart. The Gowdy T-3 cosmologies revisited. Classical Quant. Grav., 15:1581-1593, 1998.

[19] R. Iacono, M.V. Struglia, and C. Ronchi. Spontaneous formation of equatorial jets in freely decaying shallow water turbulence. Physics of Fluids, 11:12721274, 1999.

[20] J. Kevorkian. Partial Differential Equations: Analytic Solution Techniques. Springer-Verlag, New York, second edition, 2000.

[21] J.O. Langseth and R.J. LeVeque. A wave propagation method for threedimensional hyperbolic conservation laws. J. Comp. Phys., 165:126-166, 2000.

[22] R. J. LeVeque and D. H. Yong. Solitary waves in layered nonlinear media. SIAM J. Appl. Math., 63:1539-1560, 2003. 
[23] R.J. LeVeque. High-resolution conservative algorithms for advection in incompressible flow. SIAM J. Numer. Anal., 33:627-665, 1996.

[24] R.J. LeVeque. Wave propagation algorithms for multi-dimensional hyperbolic systems. J. Comp. Phys., 131:327-335, 1997.

[25] R.J. LeVeque. Balancing source terms and flux gradients in high-resolution Godunov methods: the quasi-steady wave-propagation algorithm. J. Comp. Phys., 146:346-365, 1998.

[26] R.J. LeVeque. Finite volume methods for nonlinear elasticity in heterogeneous media. In Proceedings of the ICFD conference on numerical methods for fluid dynamics, 2001.

[27] R.J. LeVeque. Finite Volume Methods for Hyperbolic Problems. Cambridge University Press, 2002.

[28] R.J. LeVeque and M. Pelanti. A class of approximate Riemann solvers and their relation to relaxation schemes. J. Comp. Phys., 172:572-591, 2001.

[29] J.M. Martí and E. Müller. Numerical hydrodynamics in special relativity. Living Rev. in Rel., 1999.

[30] J. McGregor. Semi-Lagrangian advection on conformal cubic grids. Monthly Weather Review, 124:1311-1322, 1996.

[31] C.W. Misner, K.S. Thorne, and J.A. Wheeler. Gravitation. W.H. Freeman, San Fransisco, 1973.

[32] J.A. Pons, J.A. Font, J.M. Ibáñez, J.M. Martí, and J.A. Miralles. General relativistic hydrodynamics with special relativistic Riemann solvers. Astronomy and Astrophysics, 339:638-642, 1998.

[33] M. Rancic, R.J. Purser, and F. Messinger. A global shallow-water model using an expanded spherical cube: Gnonomic versus conformal coordinates. Q.J.R. Meteorol. Soc., 122:959-982, 1996.

[34] C. Ronchi, R. Iacono, and P.S. Paolucci. The 'cubed sphere': a new method for the solution of partial differential equations in spherical geometry. J. Comp. Phys., 124:93-114, 1996.

[35] J.A. Rossmanith. A wave propagation method with constrained transport for ideal and shallow water magnetohydrodynamics. $\mathrm{PhD}$ thesis, University of Washington, Seattle, Washington, 2002.

[36] V.V. Rusanov. Calculation of interaction of non-steady shock waves with obstacles. J. Comp. Math. Phys. USSR, 1:267-279, 1961.

[37] R. Sadourny. Conservative finite-difference approximations of the primitive equations on quasi-uniform spherical grids. Monthly Weather Review, 100:211$224,1972$. 
[38] D.A. Schecter, J.F. Boyd, and P.A. Gilman. "Shallow-water" magnetohydrodynamic waves in the solar tachocline. Astrophys. J., 551:L185-L188, 2001.

[39] H. De Sterck. Multi-dimensional upwind constrained transport on unstructured grids for "shallow water" magnetohydrodynamics. In AIAA 2001-2623, June 2001.

[40] P.N. Swarztrauber, D.L. Williamson, and J.B. Drake. The Cartesian grid method for solving partial differential equations in spherical geometry. Dyn. Atmos. Oceans, 27:679-706, 1998.

[41] J.F. Thompson, B. Soni, and N.P. Weatherrill. Handbook of Grid Generation. CRC Press, 1998.

[42] Y. Tsukahara, N. Nakaso, H. Cho, and K. Yamanaka. Observation of diffractionfree propagation of surface acoustic waves around a homogeneous isotropic solid sphere. Applied Physics Letters, 77:2926-2928, 2000.

[43] M. Vinokur. Conservation equations of gasdynamics in curvilinear coordinates. J. Comp. Phys., 14:105-125, 1974.

[44] D.L. Williamson, J.B. Drake, J.J. Hack, R. Jakob, and P.N. Swartztrauber. A standard test set for numerical approximations to the shallow water equations in spherical geometry. J. Comp. Phys., 102:211-224, 1994. 

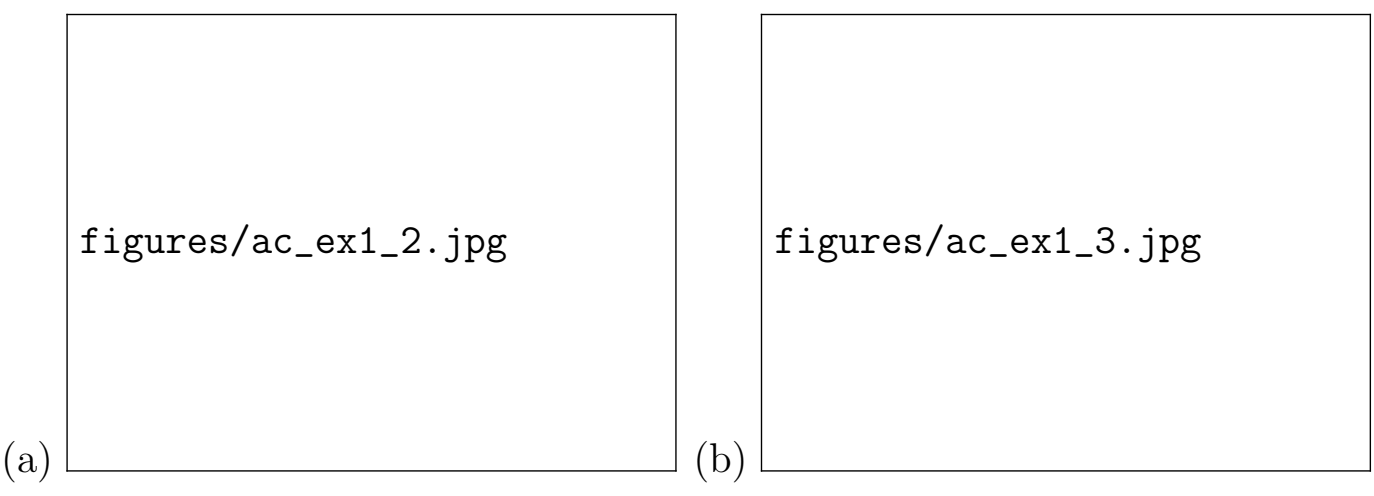

Fig. 1. The solution of the scalar field equation on a curved manifold with radially symmetric initial conditions. Shown are scatter plots of (a) the pressure and (b) the magnitude of velocity on a $100 \times 100$ grid along with a highly resolved $(6000$ points) 1D solution. This computation was run with a CFL number of 0.95 and the Monotonized Centered limiter.

(a)
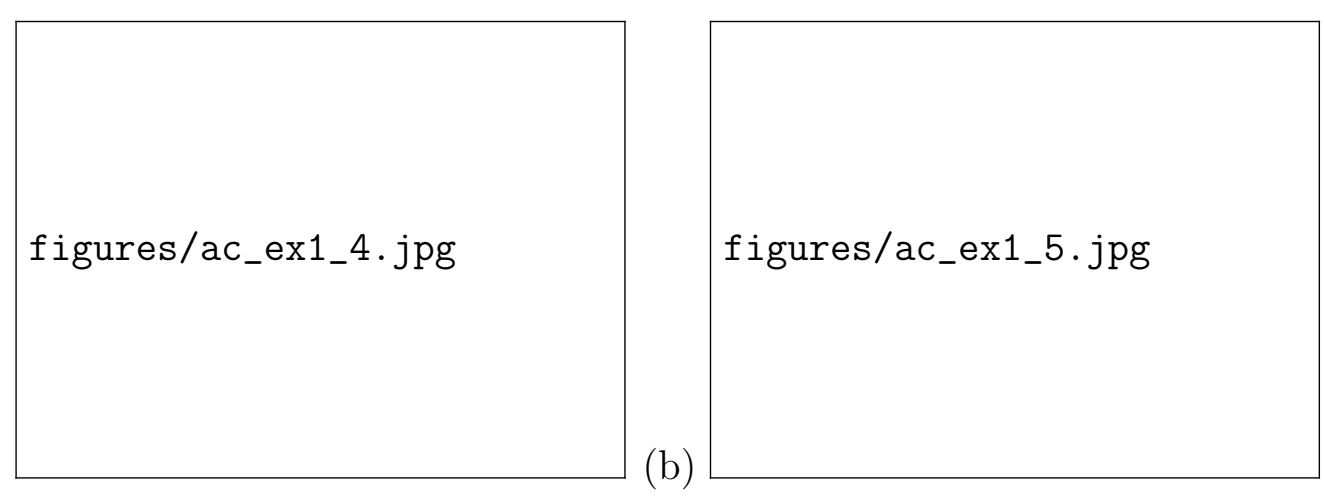

Fig. 2. Convergence study for the scalar field equations on a curved manifold. Shown are the 1-norm errors in the (a) pressure and (b) the magnitude of the three-dimensional velocity vector. 

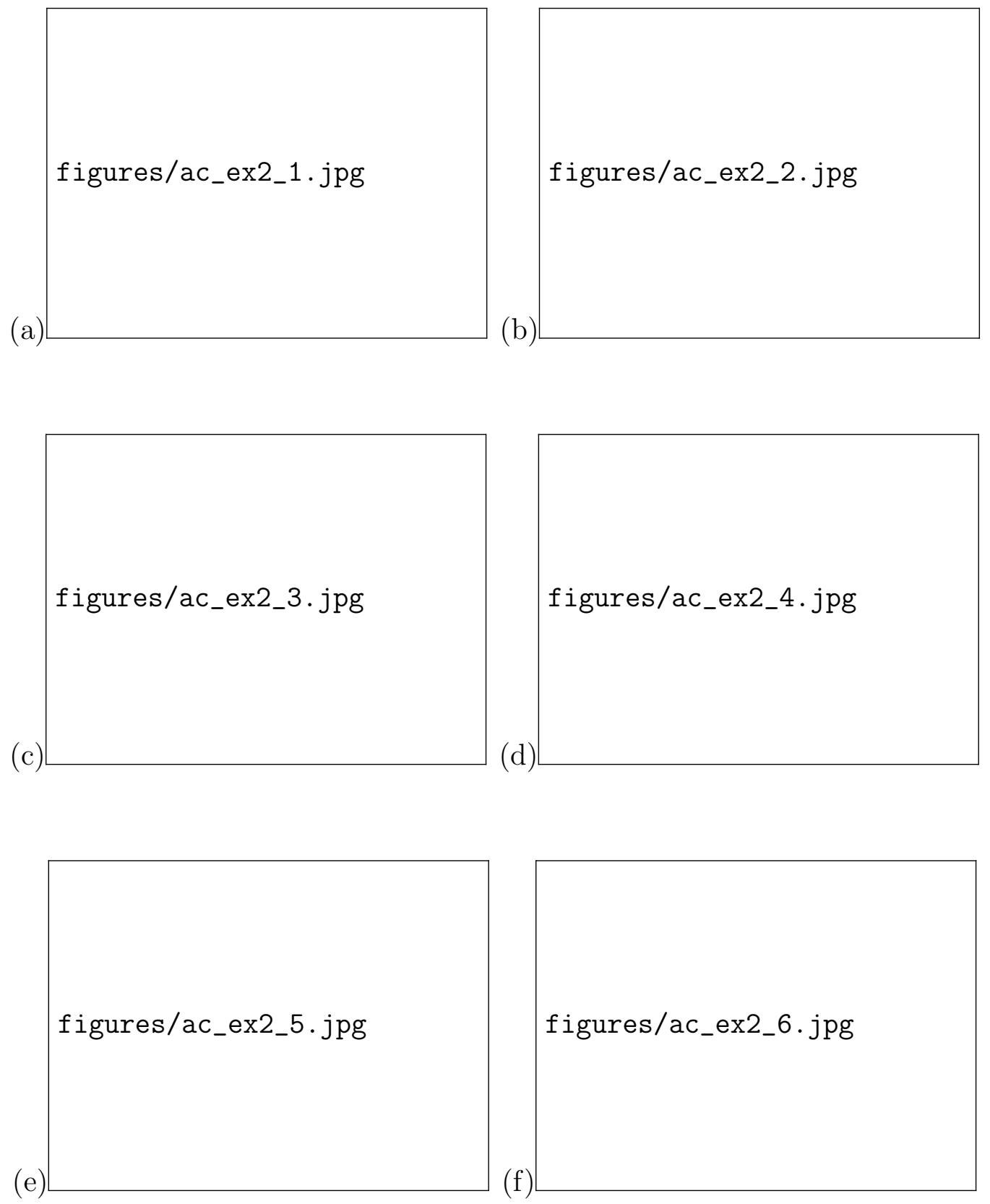

Fig. 3. Solution to the acoustic equations on a curved manifold for an initially right-going, discontinuous pressure pulse. The solution was computed on a $200 \times 200$ grid. The contours of the pressure are plotted on a plane projected down from the manifold. The manifold is also displayed but at a lower resolution than the computed solution $(50 \times 50)$. This computation was run with a CFL number of 0.95 and the Monotonized Centered limiter. 

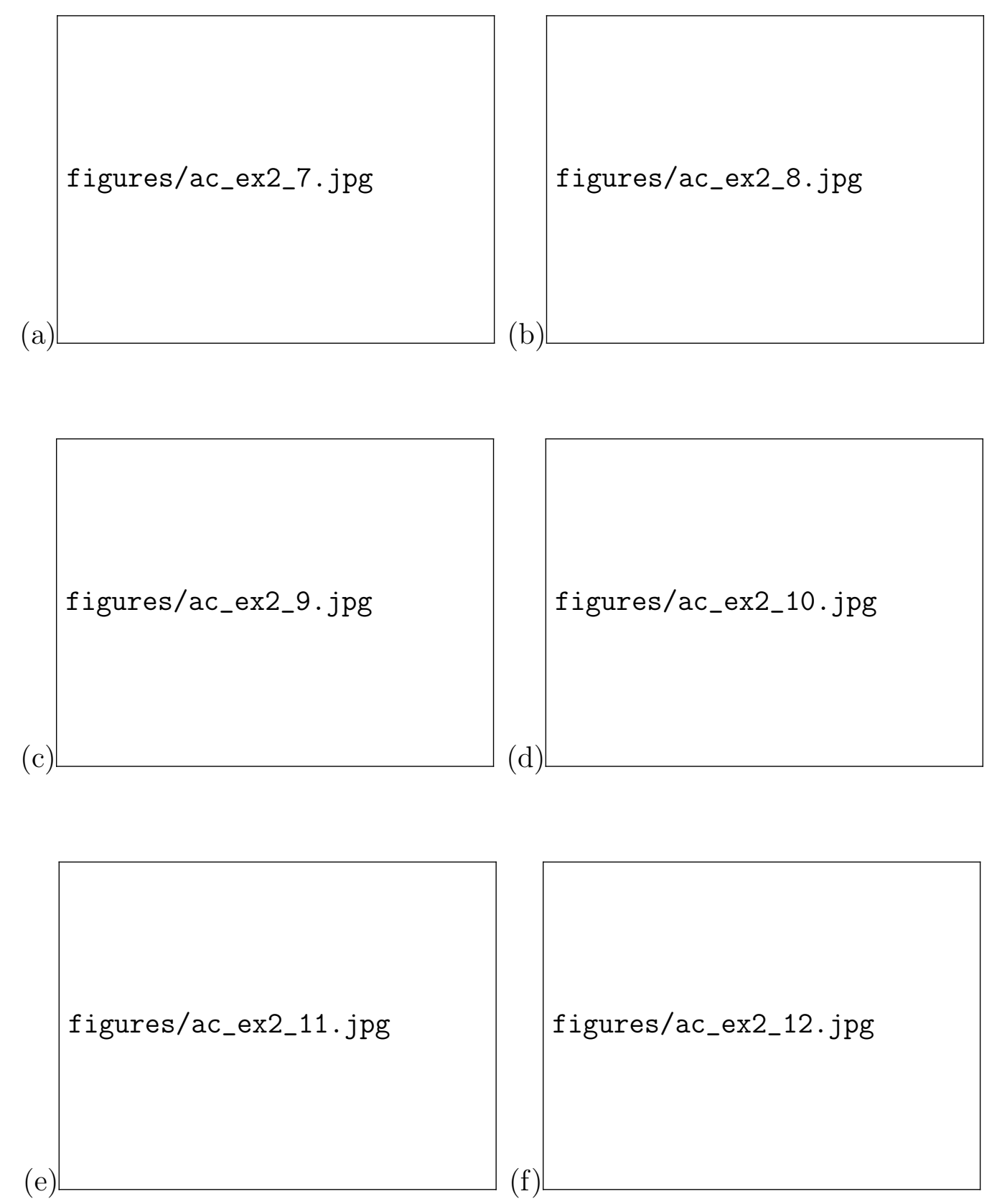

Fig. 4. Fine grid $(400 \times 400$ points $)$ calculation of the problem shown in Figure 3 . The manifold is again displayed at a resolution of $50 \times 50$ grid points. 

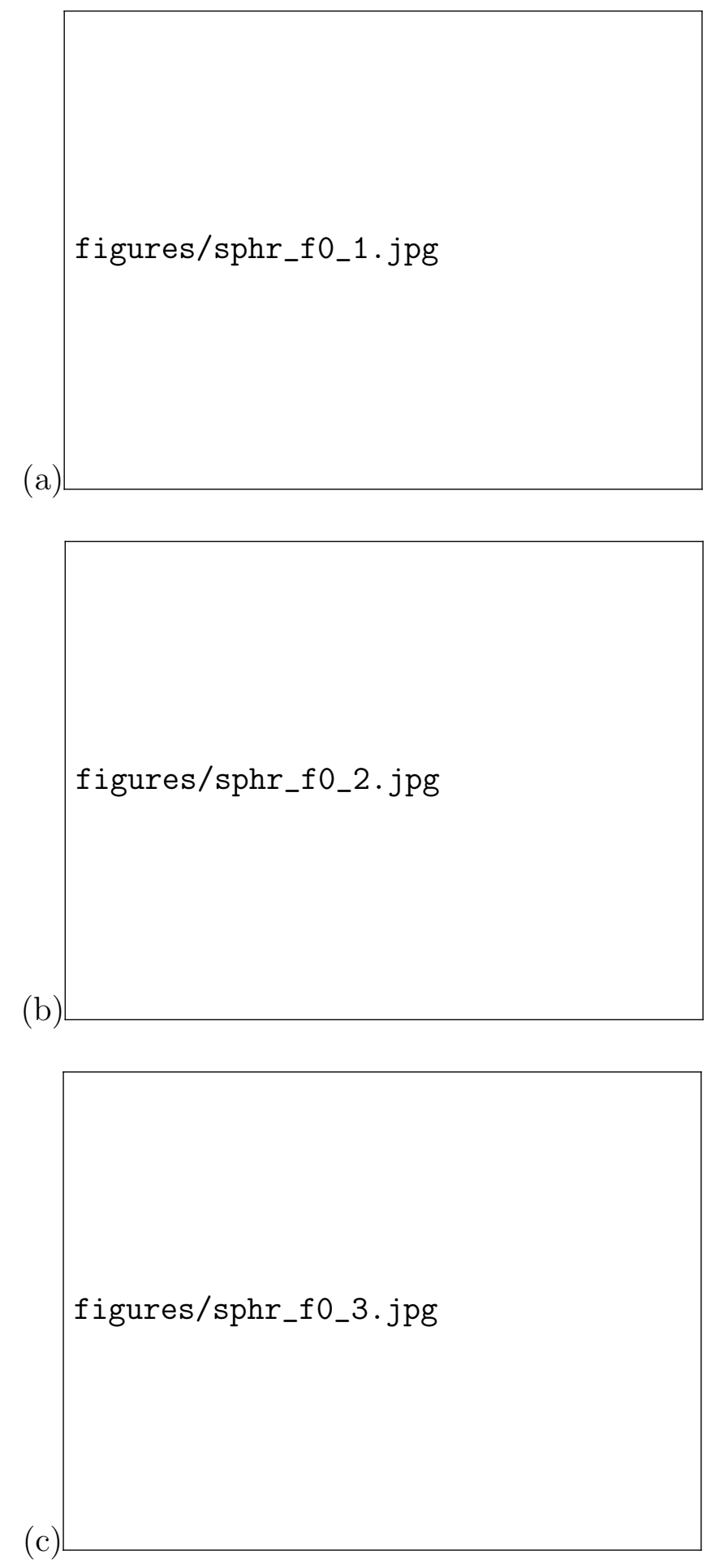

Fig. 5. Solution to the shallow water equations computed on a longitude-latitude grid in the domain $\left[-90^{\circ}, 90^{\circ}\right] \times\left[-75^{\circ}, 75^{\circ}\right]$ with $204 \times 170$ points. The solution is shown at times (a) $t=0.3$, (b) $t=0.6$, and (c) $t=0.9$. The contours do not appear circular because the sphere has been projected down to a plane. In Figure 6 we show that the solution remains symmetric. This computation was run with a CFL number of 0.95 and the Monotonized Centered limiter. 

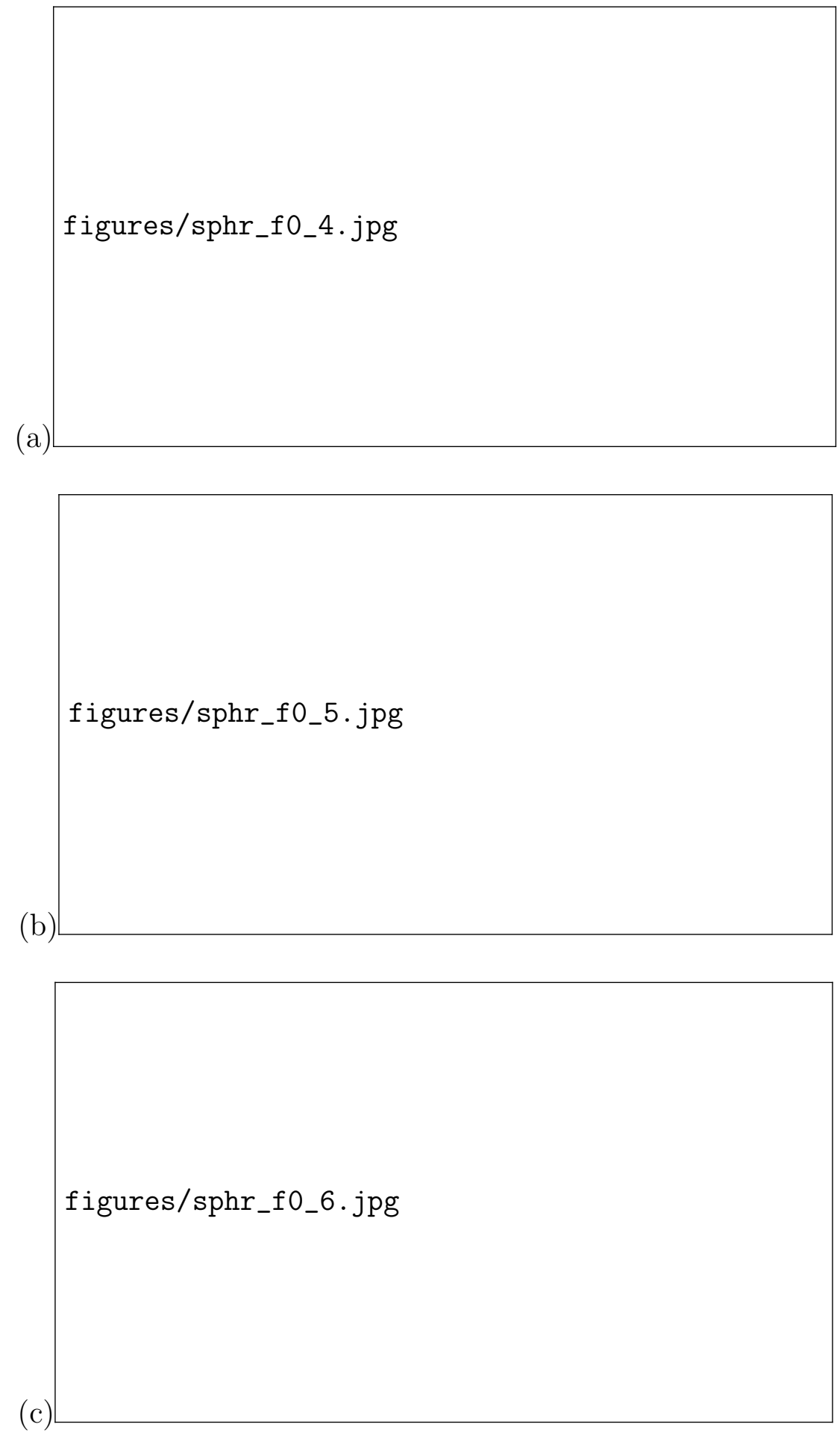

Fig. 6. Scatter plot of the solution shown in Figure 5. The curved manifold solution is plotted against a highly resolved one-dimensional symmetric solution (2000 points). The solution is again shown at times (a) $t=0.3$, (b) $t=0.6$, and (c) $t=0.9$. This computation was run with a CFL number of 0.95 and the Monotonized Centered limiter. 

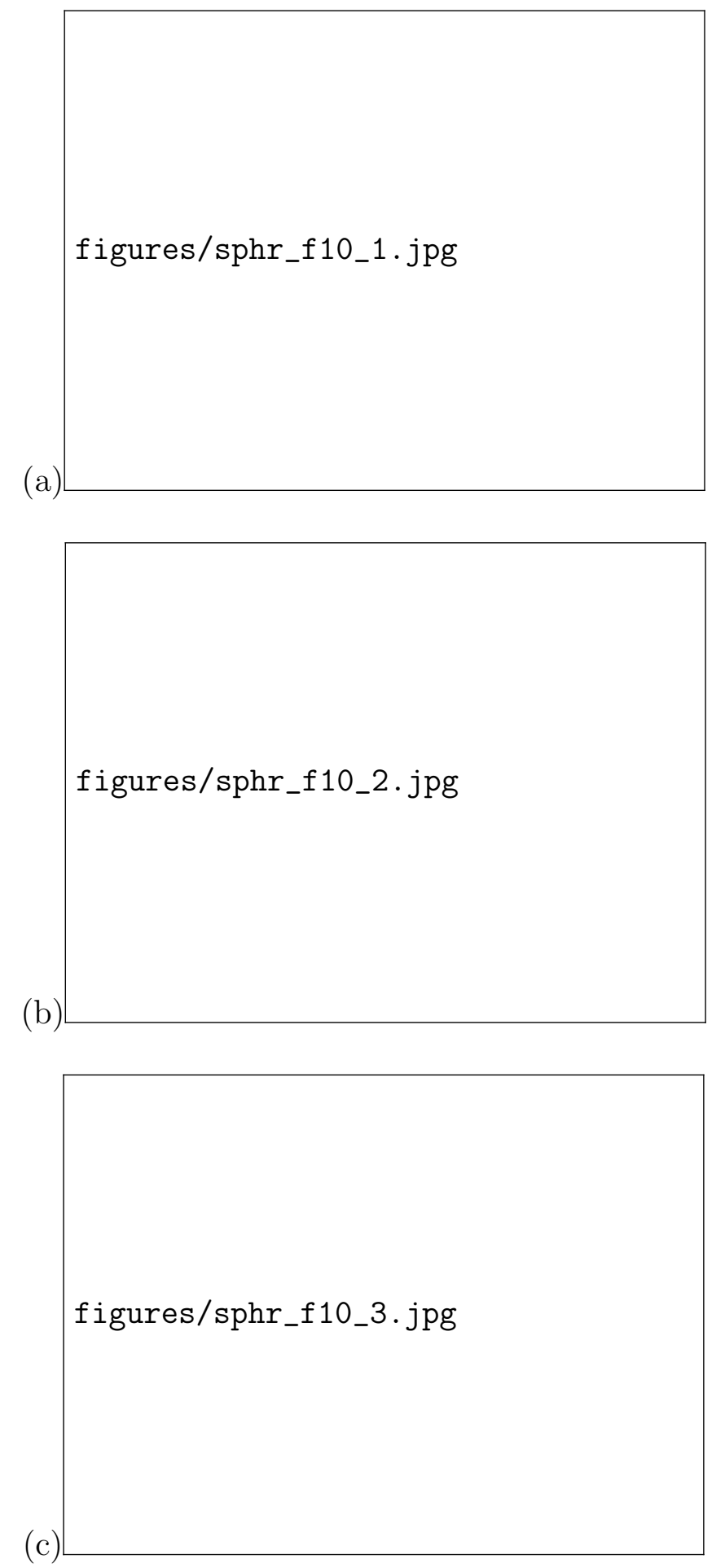

Fig. 7. Solution to the rotating shallow water equations computed on a longitude-latitude grid in the domain $\left[-90^{\circ}, 90^{\circ}\right] \times\left[-75^{\circ}, 75^{\circ}\right]$ with $204 \times 170$ points. The Coriolis parameter is set to $f=10$. The solution is shown at times (a) $t=0.4$, (b) $t=0.8$, and (c) $t=1.2$. This computation was run with a CFL number of 0.95 and the Monotonized Centered limiter. 

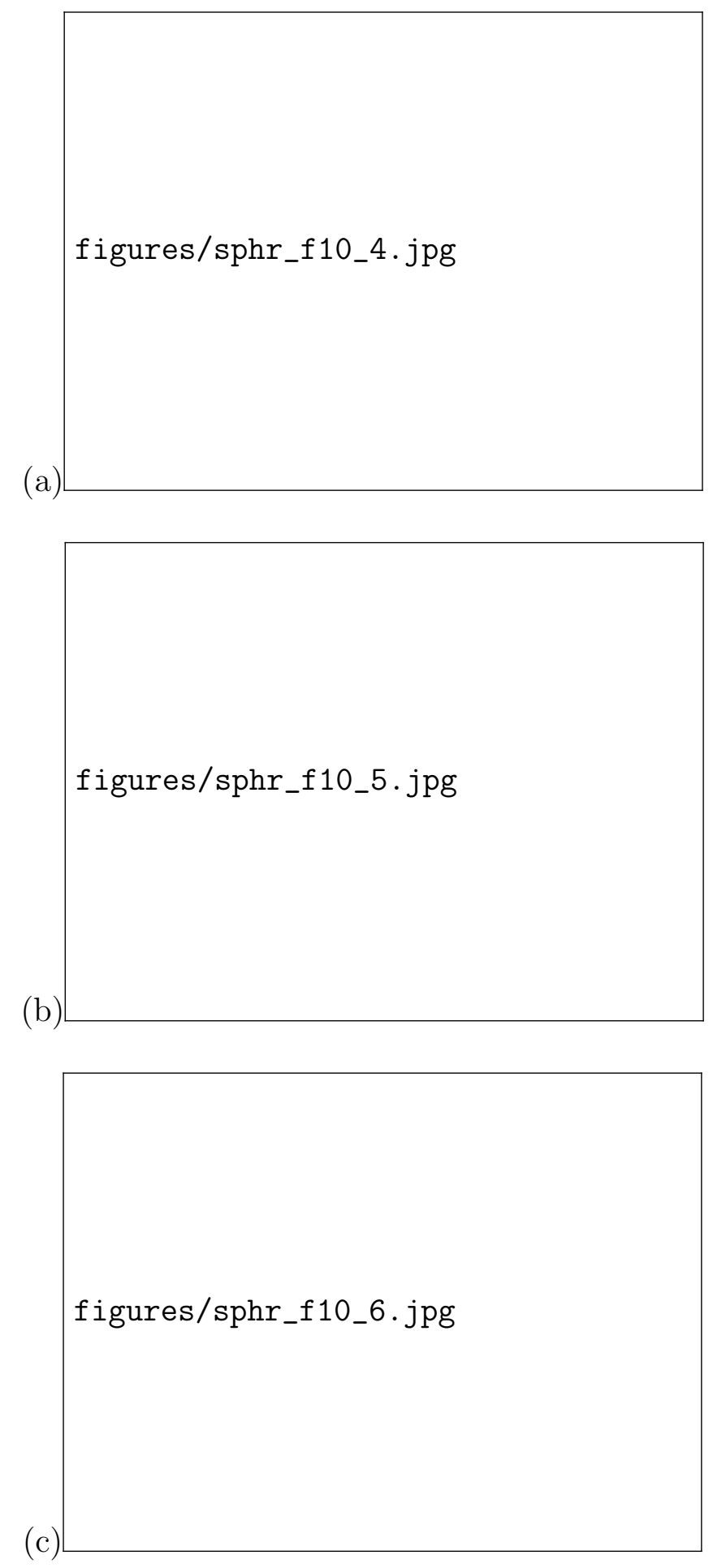

Fig. 8. Fine grid $(408 \times 340$ points $)$ calculation of the problem shown in Figure 7 . The solution is again shown at times (a) $t=0.4$, (b) $t=0.8$, and (c) $t=1.2$. 